\title{
Statistical characterization of short wind waves from stereo images of the sea surface
}

\author{
A. S. Mironov, ${ }^{1,2}$ M. V. Yurovskaya, ${ }^{3}$ V. A. Dulov, ${ }^{3}$ D. Hauser, ${ }^{1}$ and C. A. Guérin ${ }^{4,5}$ \\ Received 29 December 2011; revised 22 October 2012; accepted 23 October 2012; published 7 December 2012.
}

[1] We propose a methodology to extract short-scale statistical characteristics of the sea surface topography by means of stereo image reconstruction. The possibilities and limitations of the technique are discussed and tested on a data set acquired from an oceanographic platform at the Black Sea. A validation is made with simultaneous in situ measurements as well as results from the literature. We show that one- and two-point properties of the short-scale roughness can be well estimated without resorting to an interpolation procedure or an underlying surface model. We obtain the first cumulants of the probability distribution of small-scale elevations and slopes as well as related structure functions. We derive an empirical parametrization for the skewness function that is of primary importance in analytical scattering models from the sea surface.

Citation: Mironov, A. S., M. V. Yurovskaya, V. A. Dulov, D. Hauser, and C. A. Guérin (2012), Statistical characterization of short wind waves from stereo images of the sea surface, J. Geophys. Res., 117, C00J35, doi:10.1029/2011JC007860.

\section{Introduction}

[2] With the ever increasing accuracy of satellite microwave radar sensors for geophysical purposes and the progress of the electromagnetic wave interaction models, there is an increasing need for an accurate description of ocean short waves in various natural conditions. Two-point characteristic functions of the sea surface topography involved in the classical scattering models [see, e.g., Voronovich, 1994] are still based on model considerations rather than direct in situ measurements. In spite of obvious progresses in wave tank measurements such as reported by Zhang and Cox [1994]; Jähne et al. [2005]; Zappa et al. [2008]; and Caulliez and Guérin [2012], among others, direct in situ estimation of the small-scale topography of the sea surface is still a challenging issue. Moreover, in view of a statistical characterization it is preferable to rely on direct measurements of the topography rather than resorting to additional a priori assumptions.

[3] The spatial properties of the sea surface are routinely characterized by indirect means such as temporal measurements at a fixed location (gauge, buoys, laser) or remote sensing techniques. Detailed survey of these methods given recently in Zappa et al. [2008] shows their shortcomings.

\footnotetext{
${ }^{1}$ Université de Versailles Saint-Quentin en Yvelines, UPMC, CNRS, LATMOS, Guyancourt, France.

${ }^{2}$ Laboratoire d'Océanographie Spatiale, Ifremer, Plouzané, France.

${ }^{3}$ Marine Hydrophysical Institute, Sevastopol, Ukraine.

${ }^{4}$ Aix-Marseille Université, CNRS/INSU, IRD, MIO, UM 110, Marseille, France.

${ }^{5}$ Université de Toulon, CNRS/INSU, IRD, MIO, UM 110, La Garde, France.

Corresponding author: A. S. Mironov, Laboratoire d'Océanographie Spatiale, Ifremer, BP70, FR-29280 Plouzané, France.

(alexey.mironov@ifremer.fr)

(C2012. American Geophysical Union. All Rights Reserved. 0148-0227/12/2011JC007860
}

Contact measurements by means of wave gauges lead to the variation in time but not in space of the elevations. Radar remote sensing inversion involves a scattering model. Scanning lasers can provide the instantaneous high-resolution field of slopes but are mainly operated in wave tanks. Airborne or spaceborne lidar can be used to measure the slope vector but are restricted to a profile along the track. Recently, promising methods of polarimetric imaging [Zappa et al., 2008] and a phase-resolving spatial reconstruction technique based on a Flash Lidar Camera [Grilli et al., 2011] were proposed but are still at the stage of first publications.

[4] Stereo imaging reconstruction is well suited to assess the statistical characteristics of short waves in natural conditions. Classical utilization of this remote sensing technique does not require an underlying model for the sea surface. This technique has a long history [Cote et al., 1960; Holthuijsen, 1983; Banner et al., 1989; Shemdin et al., 1988] and has now been developed to a robust and powerful experimental tool that can be used for regular measurements of oceanic sea state dynamics and sea surface statistical properties. In particular, existing binocular and trinocular systems have been adopted for the observation of the coastal and surf zone [Wanek and Wu, 2006; Bechle and Wu, 2011; de Vries et al., 2011] as well as for offshore conditions [Gallego et al., 2011a; Kosnik and Dulov, 2011; Gallego et al., 2011b; Fedele et al., 2011, 2012; Benetazzo et al., 2012]. In these recent works the classical stereo reconstruction algorithm has been improved using various (both explicit and implicit) additional assumptions on the statistical nature of the sea surface and their brightness distribution. For example, temporal continuity of the surface is commonly implied in processing of continuous video recordings [e.g., Benetazzo, 2006]; smoothness of the surface and their brightness field is essential for the application of the variational method introduced in Gallego et al. [2011a]; a sea 
brightness model is needed for the extension of the available range of wavelength (see the brightness-based spectrum estimates by Kosnik and Dulov [2011]); a special kind of brightness field correlation must be adopted in using the subpixel methods [e.g., Wanek and $W u, 2006$; Bechle and $W u$, 2011; Benetazzo et al., 2012].

[5] However, the processing of stereo data still raises technical issues when it comes to the estimation of key statistical parameters for short waves. First, the aforementioned assumptions may appear to be incorrect for the small-scale motions of the sea surface. At least for the first step in learning the small-scale statistic properties it is interesting to apply only classical stereo reconstruction technique rejecting the additional assumptions. Second, modern devices do not cover the very wide dynamical range that is needed for the observation of surface waves at all scales varying from about $100 \mathrm{~m}$ down to $1 \mathrm{~mm}$. Stereo systems aimed at monitoring the long waves (for example, the WASS-system [Gallego et al., 2011a; Fedele et al., 2011; Benetazzo et al., 2012; Fedele et al., 2012]) enables to evaluate the mean level and the mean slope of water because these values are physically determined by the long waves. However, considering short waves only, we cannot obtain precisely these statistical values because long waves are poorly visible in the relatively small scene of observation. For such cases, when the need is to study the fluctuations with respect to a background of large-scale motion, Kolmogorov introduced the structure functions [see, e.g., Doob, 1953; Monin and Yaglom, 1999; Ishimaru, 1999]. The use of the structure functions minimizes the contribution of large-scale motion and provides us with an approach to learn the statistical properties of short waves at the sea surface without precise knowledge of the mean level and slope. Last, the consideration of the shortest waves in field conditions rises an additional difficulty [Kosnik and Dulov, 2011]. The central problem of the stereo reconstruction is to find and localize pairs of corresponding points that are images of the same object in two snapshots of the water surface made from different views. Once the corresponding points are found, the surface topography can be recovered using standard procedures [see, e.g., Benetazzo, 2006]. In the laboratory, objects can be artificially introduced on the water surface to facilitate the matching of points [Tsubaki and Fujita, 2005]. In field conditions, the only suitable objects are the brightness variations induced by surface waves. In practice, the recovery of the surface topography of waves at a given scale requires the detection of brightness variations due to smaller scale waves. This fact is the principal constraint for the spatial resolution of the stereobased method. In particular, the shortest waves cannot be recovered because there are no smaller objects on the sea surface. Sharply defined texture (capillary ripples on the sea surface) does not exist everywhere and by all weather conditions. Smooth areas without notable markers cannot be used in processing and this results in gaps in the reconstructed elevation maps [Benetazzo, 2006; Kosnik and Dulov, 2011]. While this problem does not play a significant role in dealing with well-developed large-scale stereo reconstruction, it requires a special attention in our work.

[6] The aim of this paper is to present a general methodology and some first results on the statistical characterization of ocean short wave fields. The main strength of the technique is that it does not require neither a priory assumptions for the sea surface nor interpolation procedure to compensate for the lack of sampling points. We will use high-quality data sets for three wind speeds $(7,10,15 \mathrm{~m} / \mathrm{s})$ first presented by Kosnik and Dulov [2011]. The data were processed to obtain statistically independent sea surface elevation fields using classical stereo reconstruction algorithm with improved method of searching corresponding points [Kosnik and Dulov, 2011].

[7] The paper is organized as follows. The theoretical framework of the statistical description of the sea surface is recalled in section 2 . The experimental set-up is rapidly described in section 3 and the major technical issues raised by the processing of the acquired data sets are identified and discussed in section 4. We show (section 5) that the distribution of elevations of the small-scale process can be correctly evaluated from the stereo data. The obtained distributions for different sea states can be meaningfully compared with wave gauges measurements after filtering out the large scale components. The retrieval of the sea surface slopes (section 6) requires a similar detrending procedure as well as an extrapolation procedure to compensate for the limited small-scale resolution. The estimated distributions are compared with historical [Cox and Munk, 1954] and more recent airborne measurements [Vandemark et al., 2004] by optical means. In addition to the slopes, the distribution of chords at the surface can be derived and successively compared with gauge wire as well as airborne [Vandemark et al., 2004] measurements. The two-points properties of the small-scale roughness can be characterized in the same manner. We show (section 8) that the autocorrelation function of the small-scale process and its Fourier transform are consistent with alternative measurements of the wave spectrum. Our last result (section 9) concerns the derivation of the skewness function that has an important meaning in remote sensing theories and witnesses for the asymmetric nature of waves. We derive it experimentally and provide an original and accurate parametrization of this otherwise unknown function.

\section{Theoretical Background}

\subsection{Distribution of Sea Surface Elevations}

[8] The one-point distribution of elevations on the sea surface is the primary and simplest quantity to extract. Statistical properties of sea surface elevations under different environmental conditions are well documented by various experimental and theoretical investigations (see, e.g., Longuet-Higgins et al. [1963]; Huang et al. [1983]; and Tayfun and Fedele [2007] for a few milestones). Under a mere Gaussian assumption, the field of elevations is characterized only by the root mean square of elevation $(\sigma)$ or significant wave height $\left(H_{s}=4 \sigma\right)$. However, nonlinear corrections are necessary to reproduce the typical Stokes wave form of gravity waves. They are usually limited to the thirdand fourth-order renormalized cumulants, namely the skewness $\left(\lambda_{3}\right)$ and excess kurtosis $\left(\lambda_{4}\right)$ coefficients, which are necessary to take into account the vertical asymmetry of waves and the occurrence of flat troughs. They are defined, respectively, by:

$$
\sigma^{2}=\left\langle\eta^{2}\right\rangle, \sigma^{3} \lambda_{3}=\left\langle\eta^{3}\right\rangle, \sigma^{4} \lambda_{4}=\left\langle\eta^{4}\right\rangle-3\left\langle\eta^{2}\right\rangle^{2}
$$


where $\eta$ is the centered elevation and the bracket $\langle\cdot\rangle$ denotes the ensemble average.

\subsection{Distribution of Sea Surface Slopes}

[9] The mean square slope (mss) is a fundamental quantity for the description of the sea surface. It is meaningful in terms of sea state and flux exchanges in the upper-layer and, at the same time, is one of the primary parameters that can be inverted from remote sensing techniques. We recall that the mss is the variance of the slope vector $\nabla \eta=\left(\eta_{x}, \eta_{y}\right)$ :

$$
\mathrm{mss}=\left\langle|\nabla \eta|^{2}\right\rangle=\left\langle\eta_{x}^{2}\right\rangle+\left\langle\eta_{y}^{2}\right\rangle=\sigma_{x}^{2}+\sigma_{y}^{2}
$$

with obvious notations. It can also be obtained from the second-moment of the omnidirectional spectrum (see the discussion in section 8).

[10] The mss in open sea conditions has been well characterized since the historical optical airborne measurements of Cox and Munk [1954], later confirmed by spaceborne measurements [Bréon and Henriot, 2006]. The deviation from the two-dimensional Gaussian distribution is originally quantified in terms of Gram-Charlier coefficients, which are a generalization of the one-dimensional cumulants [Cox and Munk, 1956]. The skewness coefficients $\left(C_{i j}\right)$ are defined by:

$$
\sigma_{x}^{i} \sigma_{y}^{j} C_{i j}=\left\langle\eta_{x}^{i} \eta_{y}^{j}\right\rangle, \quad i, j \geq 0,
$$

with $i+j=3$. The kurtosis coefficients are defined by the same equation with $i+j=4$.

\subsection{Structure Functions and Chords}

[11] Structure functions can be interpreted as the moments of chords, which are segments joining two points of the surface at a given horizontal distance. They are defined by:

$$
S_{n}(\boldsymbol{r})=\left\langle(\eta(\boldsymbol{r})-\eta(\mathbf{0}))^{n}\right\rangle
$$

for every lag vector $\boldsymbol{r}$. The renormalized structure functions,

$$
\frac{S_{2}(\boldsymbol{r})}{r^{2}}, \frac{S_{3}(\boldsymbol{r})}{S_{2}^{3 / 2}(\boldsymbol{r})}, \frac{S_{4}(\boldsymbol{r})}{S_{2}^{2}(\boldsymbol{r})}, \ldots
$$

are the directional mss, skewness, kurtosis, etc., of the slopes of chords. The slopes of chords of given length $L$ can be thought as the slopes of low-pass filtered surface, where wavelengths smaller than the scale $L$ have been removed. It is thus related to a filtered mss, obtained by calculating the second moment of a truncated spectrum.

[12] We denote by omnidirectional mss and kurtosis the angular averaged directional quantities (the omnidirectional skewness vanishes by symmetry). Similarly we define the omnidirectional structure function:

$$
S_{n}(r)=\frac{1}{2 \pi} \int_{0}^{2 \pi} d \theta S_{n}(r, \theta)
$$

where $\theta$ is the azimuth angle of the vector $\boldsymbol{r}$ with given norm $r$. We use the same notation $S(\boldsymbol{r})$ and $S(r)$ for the directional and omnidirectional quantities, the distinction being clear from their argument $(r$ or $r$ ). Note that the directionally averaged structure functions are only relevant for even-order $n$ and vanish at odd-order in view of their antisymmetry. The directionally averaged mss coincides with the omnidirectional mss. However, the omnidirectional kurtosis is different from the direction averaged kurtosis:

$$
\lambda_{4}^{o m n i}=\frac{S_{4}(r)}{S_{2}^{2}(r)} \neq \frac{1}{2 \pi} \int_{0}^{2 \pi} d \theta \frac{S_{4}(r, \theta)}{S_{2}^{2}(r, \theta)}=: \lambda_{4}^{d i r \cdot a v}
$$

\subsection{Structure Functions and Slopes}

[13] Assuming finite slopes, a first-order Taylor expansion of the structure function at the origin gives:

$$
S_{2}(\boldsymbol{r})=\sigma_{x}^{2} x^{2}+\sigma_{y}^{2} y^{2}+2 \sigma_{x y}^{2} x y
$$

The cross-term on the right-hand side vanishes after angular integration and it follows easily that:

$$
\mathrm{mss}=\lim _{r \rightarrow 0} \frac{2 S_{2}(r)}{r^{2}} .
$$

Hence the total mss can be estimated through the limiting behavior of the omnidirectional structure function at the origin. Also, by a Taylor expansion at the origin we have:

$$
\begin{aligned}
S_{3}(\boldsymbol{r}) & \simeq \sigma_{x}^{3} C_{30} x^{3}+3 \sigma_{x} \sigma_{y} x y\left(C_{21} x+C_{12} y\right)+\sigma_{y}^{3} C_{03} y^{3} \\
S_{4}(\boldsymbol{r}) & \simeq \sigma_{x}^{4} C_{40} x^{4}+4 \sigma_{x}^{3} \sigma_{y} C_{31} x^{3} y+6 \sigma_{x}^{2} \sigma_{y}^{2} C_{22} x^{2} y^{2} \\
& +4 \sigma_{x} \sigma_{y}^{3} C_{13} x y^{3}+\sigma_{y}^{4} C_{04} y^{4}
\end{aligned}
$$

so that (several terms cancel after angular integration):

$$
\lim _{r \rightarrow 0} \frac{S_{4}(r)}{r^{4}}=\frac{3}{8}\left(C_{40} \sigma_{x}^{4}+\sigma_{y}^{4} C_{04}\right)+\frac{3}{4} C_{22} \sigma_{x}^{2} \sigma_{y}^{2}
$$

We define the omnidirectional kurtosis of slopes by

$$
\mathrm{K}_{\mathrm{omni}}=\lim _{r \rightarrow 0} \frac{S_{4}(r)}{S_{2}^{2}(r)},
$$

which can be expressed in terms of the kurtosis coefficients:

$$
\mathrm{K}_{\mathrm{omni}}=3+\frac{3}{4}\left(C_{40} \frac{\sigma_{x}^{4}}{\mathrm{mss}^{2}}+\frac{\sigma_{y}^{4}}{\mathrm{mss}^{2}} C_{04}\right)+\frac{3}{2} C_{22} \frac{\sigma_{x}^{2} \sigma_{x}^{2}}{\mathrm{mss}^{2}}
$$

[14] The skewness coefficients can in principle also be estimated from the limiting behavior of the third-order structure function along one direction. For instance:

$$
C_{30}=\lim _{x \rightarrow 0} \frac{S_{3}(x, y=0)}{S_{2}^{3 / 2}(x, y=0)},
$$

This will be discussed in more detail in the last section. In the Gaussian case, note that the skewness and some of the kurtosis $\left(C_{31}, C_{13}\right)$ coefficients vanish while $C_{40}=C_{04}=3$ and $C_{22}=1$.

\subsection{Structure Functions and Remote Sensing}

[15] The precise knowledge of the structure functions of the sea surface topography is of crucial importance for remote sensing applications. Many analytical scattering models (see, e.g., Voronovich [1994] for a review) actually involve the two-point characteristic function of elevations:

$$
\Psi\left(Q_{z}, \boldsymbol{r}\right)=\left\langle\exp \left(\mathrm{Q}_{z}(\eta(\boldsymbol{r})-\eta(0))\right)\right\rangle,
$$




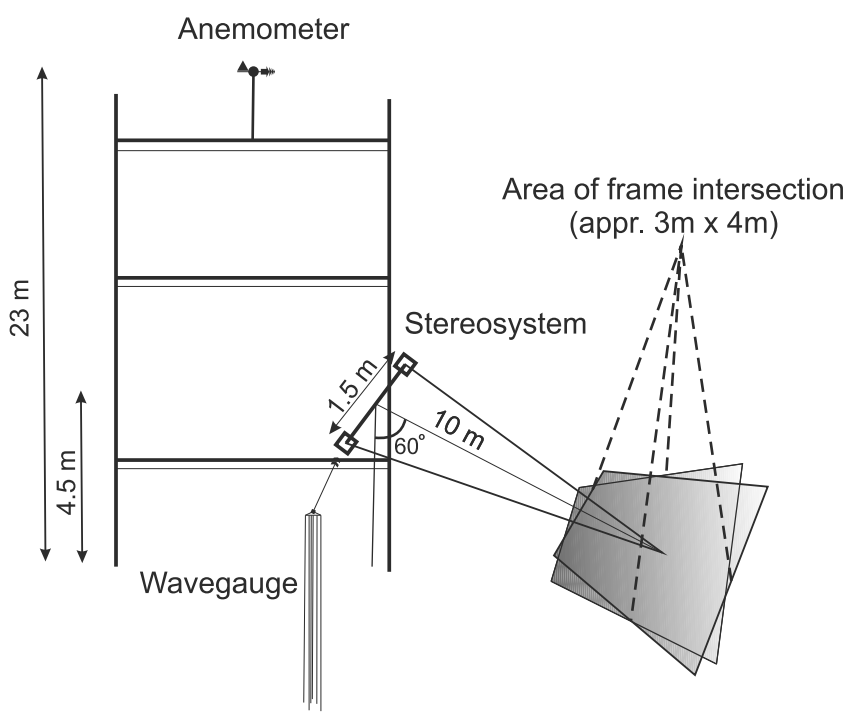

Figure 1. Experimental set-up.

where $Q_{z}=2 \mathrm{~K}_{0} \cos \phi, K_{0}$ is the electromagnetic wave number and $\phi$ the incidence angle. Under a weakly nonGaussian assumption this characteristic function can be expanded as:

$$
\Psi\left(Q_{z}, \boldsymbol{r}\right) \simeq \exp \left(-\frac{Q_{z}^{2}}{2} S_{2}-\frac{\mathrm{Q}_{\mathrm{z}}^{3}}{3 !} \mathrm{S}_{3}+\frac{\mathrm{Q}_{\mathrm{z}}^{4}}{4 !}\left(\mathrm{S}_{4}-3 \mathrm{~S}_{2}^{2}\right)\right),
$$

where as before $S_{n}$ are the structure functions of chords (the argument $\boldsymbol{r}$ is omitted for sake of compactness). It is common to resort to a Gaussian assumption $\left(S_{3}=0\right.$ and $S_{4}=3 S_{2}^{2}$ ) to avoid introducing higher-order structure functions. However, the skewness and kurtosis functions are necessary to account for some qualitative features of remote sensing data such as upwind-downwind asymmetry [Mouche et al., 2007] and peakedness [Bringer et al., 2012] of radar observations. These functions are in general unknown except at the origin, from which they are usually extrapolated. Choosing the $x$ axis along the wind direction, assuming crosswind symmetry and making use of the following relation (whose derivation is justified in McDaniel [2003]):

$$
C_{12} \sigma_{y}^{2}=\frac{1}{3} C_{30} \sigma_{x}^{2}
$$

we obtain from the Taylor expansion (10):

$$
\begin{aligned}
S_{3}(\boldsymbol{r}) & =x \sigma_{x}\left(C_{30} \sigma_{x}^{2} x^{2}+3 C_{12} \sigma_{y}^{2} y^{2}\right) \\
& =\frac{1}{3} x \sigma_{x}^{3} C_{30}\left(x^{2}+y^{2}\right)=\frac{1}{3} r^{3} \sigma_{x}^{3} C_{30} \cos \theta,
\end{aligned}
$$

where $\theta$ is the azimuthal angle with respect to wind. This parametrization has been used in Mouche et al. [2007] with the skewness coefficients $C_{i j}$ reported by Cox and Munk [1954]. Note that the experimental value is negative so that $S_{3}$ is negative in the upwind direction and positive in the downwind direction. To describe the skewness function at larger lags, a more general parametrization has been proposed by Chen et al. [1992] with a different azimuthal dependence and a Gaussian cut-off:

$$
S_{3}(\boldsymbol{r})=\left(\frac{r \cos (\theta)}{s_{0}}\right)^{3} \exp \left\{-\left(\frac{r}{s_{0}}\right)^{2}\right\} .
$$

Here the parameter $s_{0}$ is an effective distance that can be tuned in the model. The present set of stereo data makes it possible to test this parametrization. As we will show in section 9, a different functional form must be introduced to be consistent with the observations.

\section{Experimental Set-Up}

[16] The experiment was conducted in October 2009 on the Black Sea platform of Marine Hydrophysical Institute (Crimea, Ukraine). The platform is located 500 meters off the coast by a depth of about $30 \mathrm{~m}$. Stereo images were acquired by means of two synchronized cameras with $72 \mathrm{~mm}$ focal length situated at $4.5 \mathrm{~m}$ above the sea level with grazing angle of $30^{\circ}$. The cameras were set about 1.5 meter apart (see Figure 1). Stereo system geometry and camera parameters were devised to get the shortest wave extraction and allowed to obtain 16 bit images with $1 \mathrm{~mm}$ spatial resolution. The fundamental point here is the small exposure time $(1 / 1000 \mathrm{~s})$ and the small synchronization time of the cameras (better than $0.1 \mathrm{~ms}$ ). For longer times the shortest waves, which are carried by the orbital velocities of long waves are blurred in the images and become difficult to distinguish. Photographing was made every $10-15 \mathrm{~s}$ providing statistically independent realizations of small-scale sea surface topography. Sea surface spatial coordinates were obtained from collected stereo pairs of photographs with the standard approach based on epipolar geometry and pinhole camera model with distortion correction [e.g., Benetazzo, 2006] and improved homologous point search algorithm [Kosnik and Dulov, 2011]. Simultaneous measurements of temperature, wind speed and direction were recorded by the automatic meteorological station installed on the platform.

[17] Wave height measurements at $10 \mathrm{~Hz}$ sampling frequency and $2 \mathrm{~mm}$ accuracy were performed with six resistant wave gauges. These gauges were used to record 20 minutes time series during each experimental measurement. Because of some failure of equipment during the experiment, four gauges did not work properly in case 2 and were discarded. Therefore the distribution obtained in this case is more noisy.

[18] Table 1 lists the main environmental parameters for the different experimental conditions. Each series of frames

Table 1. Experimental Conditions

\begin{tabular}{lccc}
\hline & \multicolumn{3}{c}{ Case Number } \\
\cline { 2 - 4 } & 1 & 2 & 3 \\
\hline Day of October, 2009 & 28 & 7 & 19 \\
Wind speed & 7 & 10 & $13-17$ \\
Wind direction & North & East & East \\
Fetch (km) & 0.5 & $\sim 400$ & $\sim 400$ \\
Presence of swell & Yes & Yes & No \\
Number of frames & 76 & 72 & 67 \\
Wave length of spectrum peak (meter) & 2.4 & 32 & 26 \\
Wave period of spectrum peak (s) & 1.3 & 4.5 & 4.1 \\
Frequency of spectrum peak (Hz) & 0.8 & 0.22 & 0.24 \\
Significant wave height (meter) & 0.3 & 0.85 & 0.9 \\
\hline
\end{tabular}


corresponds to a time span of twenty minutes. The full experimental and processing procedure is reported in Kosnik and Dulov [2011].

\section{Major Technical Issues}

[19] The natural and instrumental limitations impose some restrictions on the recoverable properties of sea surface elevation. Here we discuss the main issues raised by the processing of the current data set. The processing of images of sea surface combines several sequential stages and each of them has a certain limitation and source of error. For example, stereo photography is not applicable at low wind speeds, when the sea surface has very few markers to identify the points for correspondence in stereo pairs. In severe storm conditions the search of corresponding points also faces a difficulty due to the presence of foam that often turns out to be different on the left and right images because of the difference in camera view direction and thus the difference in light reflection from the bubbles.

[20] I1: Limited accuracy-The main errors that arise in stereo analysis include 1) calibration error, i.e. the error of spatial coordinate recovery, 2) the resolution or quantization error, 3) the typical error of corresponding pixel search and 4) the spikes on the recovered surfaces that appear due to the wrong determination of corresponding pixels in stereo pair. Let us consider these factors successively. The error of spatial coordinate recovery is determined by the accuracy of estimation of the epipolar matrix used for triangulation. The respective numerical values are calculated in the calibration procedure [Bouguet, 2004]. However, to evaluate the resulting error in our measurements and to verify the correctness of the calibration results after equipment reinstalling, some test snapshots of subjects with known linear dimensions were made in each experimental run. It was found that a significant error occurs in the direction of the stereo system axis, i.e. in determining the distance to the object. However, linear dimensions of objects at the typical distances from the camera considered in our measurements $(\sim 10 \mathrm{~m})$ are determined with the accuracy better than $1 \%$. Since the systematic error in distance determination contributes only to the low wave-number trend and does not affect the short-wave statistics, we will consider only the error of measurement of surface displacement relative to its average level, i.e. accept the calibration error of $\delta S_{\text {calib }}=1 \%$. The parallax error determines the accuracy of coordinate recovery. Benetazzo [2006] suggested the estimation of the maximum error for the $2 \mathrm{D}$ model of stereo rig geometry:

$$
\begin{aligned}
e r_{Z} & =\frac{Z^{2} \sin 2 \beta}{2 T N \cos (\beta+\alpha)^{2}} \\
e r_{X} & =\frac{Z \sin 2 \beta}{2 N \cos (\beta+\alpha)^{2}} \\
e r_{Y} & =\frac{Z \sin 2 \beta}{2 N \cos (\beta)^{2}} .
\end{aligned}
$$

[21] Here $X$ axis is parallel to the baseline, $Z$ axis is perpendicular to the baseline and lies in camera optical axes plane, $\alpha$ is the angle between the line of sight of the camera, $\beta$ is the half view angle of the camera, $T$ is the baseline and $N$ is the number of pixels of the $1 \mathrm{D}$ camera. The errors in the world coordinate system are calculated with the use of rotation matrix $\left(\boldsymbol{e} \boldsymbol{r}^{\prime}\right)=(R)(\boldsymbol{e r})$. According to these formula, the horizontal coordinate errors are $0.5 \mathrm{~mm}$ in baseline direction and $3 \mathrm{~mm}$ in perpendicular direction, and the elevation recovery error is $1 \mathrm{~mm}$. On the other hand, we can also estimate the errors for the central pixel considering that it is of order of the spatial pixel size on the surface:

$$
\begin{aligned}
& e r_{X c}=2 L \tan \left(\beta_{H} / 2\right) / N_{H} \\
& e r_{Y c}=2 L \tan \left(\beta_{V} / 2\right) /\left(N_{V} \sin \gamma\right) \\
& e r_{h c}=2 L \tan \left(\beta_{V} / 2\right) \cos \gamma / N_{V},
\end{aligned}
$$

where $\beta_{V}=11^{\circ}$ and $\beta_{H}=17^{\circ}$ are the camera horizontal and vertical angles of view, $\gamma=30^{\circ}$ is the grazing angle, $N_{H}=$ 3888 and $N_{V}=2592$ are the numbers of pixels in horizontal and vertical directions, respectively, and $L=10 \mathrm{~m}$ is the distance to the surface. We obtain $e r_{X c}=0.8 \mathrm{~mm}, e r_{Y c}=$ $1.5 \mathrm{~mm}, e r_{h c}=0.6 \mathrm{~mm}$.

[22] The correctness of the determination of corresponding points on a pair of images is more difficult to estimate. To minimize error in this process we apply several stages to filter out the "false" points in our algorithm [Kosnik and Dulov, 2011]. The difference between stereo reconstruction of sea surface and the classical problem of stereo reconstruction is in the fact that the sea surface does not possess well-selected objects. One can use only the spatial variations of sea surface brightness. This approach is quite suitable for retrieval wavelengths of $1 \mathrm{~m}$ or more but for smaller scales significant errors appear due to differences in brightness of images obtained from two different points of observation. To minimize these errors we apply the technique that was first described in Kosnik and Dulov [2011]. Briefly, it consists in several stages of image processing before correlation analysis: a) the transform of one image of stereo pair to make it coincide at large scales with the second image; b) removal of low frequencies in Fourier space in order to keep only the small objects at the sea surface that form the image texture; c) application of different approaches to filter out the "false" points at several steps of the algorithm.

[23] Nevertheless the reconstructed surfaces can still contain sporadic unphysical peaks but their number and relative amplitudes are small and their contribution to the short wave statistics is negligible. The typical accuracy of corresponding pixel search can be estimated from correlation coefficient distribution. An example of correlation function is presented in Figure 2. In general the stereo matching algorithm can be significantly improved by minimizing the correlation function width up to the subpixel scales [Wanek and Wu, 2006; Benetazzo, 2006; Bechle and $W u, 2011]$. In our case the maximum of correlation function is determined with the accuracy of 12 pixel while its half-width can be as large as 5-10 pixels. This means that the resulting errors are a few times larger than those predicted by the above formulas. Hence the maximum error on horizontal coordinates is of order of $1 \mathrm{~cm}$ and the error on elevation is about several millimeters.

[24] I2: Limited range of scales-Current experimental measurements were aimed at the study of short gravity and capillary-gravity waves. A sufficient accuracy for this analysis imposed a limited observation area of about $3 \times 4$ meters with a pixel footprint size of approximately $1 \mathrm{~mm}$. The window used for the correlation analysis in corresponding 


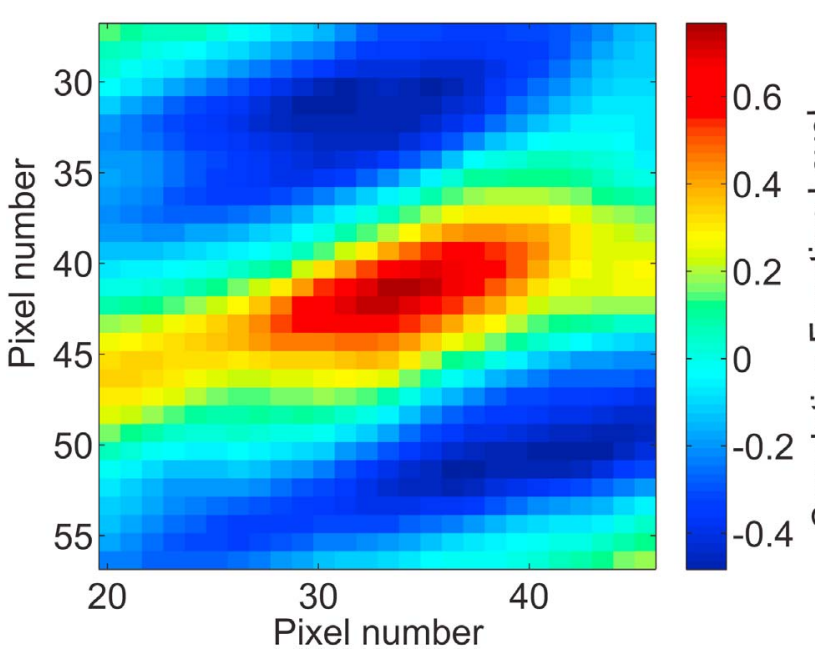

Figure 2. Example of the correlation function calculated to define corresponding points between pair of images. Window size is $30 \times 30$ pixels in this example.

pixel search contains several tens of points and the spatial resolution after stereo processing has an order of $1 \mathrm{~cm}$, excluding gaps. These low- and high-frequency cut-off limit the accessible wave numbers to an intermediate range, say $5 \mathrm{rad} \mathrm{m} \mathrm{m}^{-1}<k<200 \mathrm{rad} \mathrm{m}^{-1}$. The field of elevations recovered from each stereo pair of images is relative to the mean level over the patch, so that larger waves are only seen through their tilting and hydrodynamical modulation effect but not through their absolute amplitude.

[25] I3: The problem of the mean plane-The mean tilted plane over each image is in principle imposed by the largest waves. However, a systematic bias in the estimation of the mean plane can be introduced by errors in the stereographic reconstruction procedure. Precisely, the orientation between the camera and world coordinate systems is given by successive geometric transformations (rotations and translations) that might be poorly estimated (see Benetazzo [2006] for a complete analysis of the sources of errors).

[26] I4: Gaps in reconstructed data-At the sea surface, smooth areas without notable markers (that is in absence of smallest-scale texture) cannot be satisfactorily reconstructed. This results in gaps in reconstructed elevations (see Figure 3, right). The presence of gaps prevents the application of standard regular-grid algorithms of further data processing. But this difficulty is inherent in small-scale stereo reconstruction [Benetazzo, 2006; Kosnik and Dulov,

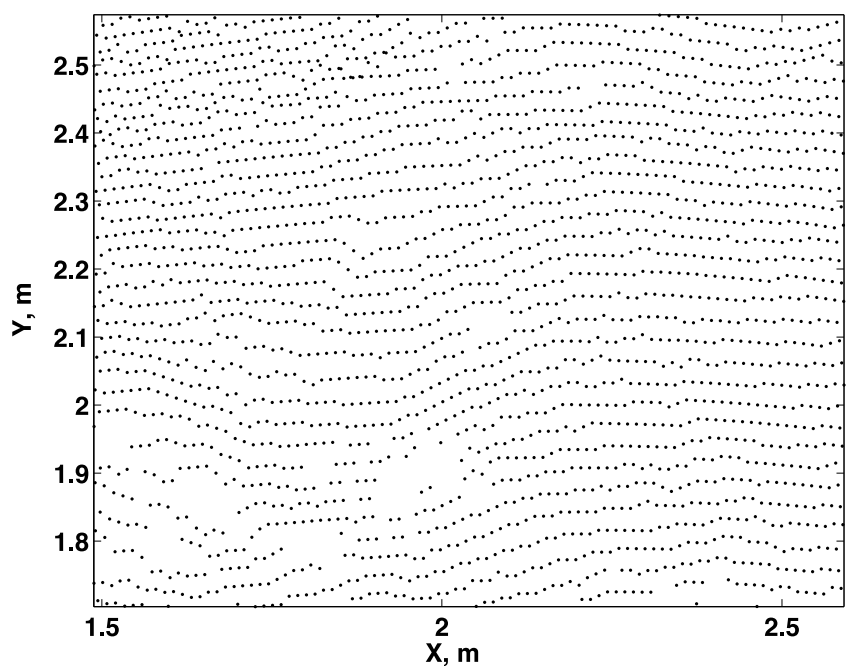

Figure 4. Available horizontal coordinates over a frame. The sampling distribution is irregular but remains close to a grid. Some "islands" with missing points are visible.

2011]. The number of retrieved elevation points for each snapshot varies from 20387 to 36235 (in average 29000 ) depending on gap locations. For the areas free of gaps, resulting space resolution of stereo reconstruction is of about $1 \mathrm{~cm}$.

[27] I5: Irregular grid-According to the specificity of the stereo-reconstruction methodology, the positions of homologous points are strongly associated with irregular ripples pattern and local elevations of sea surface. As a result, reconstructed elevations $z=\eta(x, y)$ are found on an irregular horizontal grid. A possible solution is a reinterpolation and projection on a regular grid. However, this implies implicit smoothing of the surface and also causes bias in the statistical estimation.

[28] I6: Gridding effect-The "gridding effect" is the bias introduced while estimating the finite differences on a twodimensional grid. Surface elevation points remain rather close to a regular two-dimensional grid, even after the process of identification of homologous points on images of stereo pair. The estimation of the directional slope via two nearest neighbors on a grid is strongly biased by the varying distance between points, according to spatial relative disposition of points: on a diagonal or parallel to the elementary cell. Figure 4 displays an example of the available horizontal
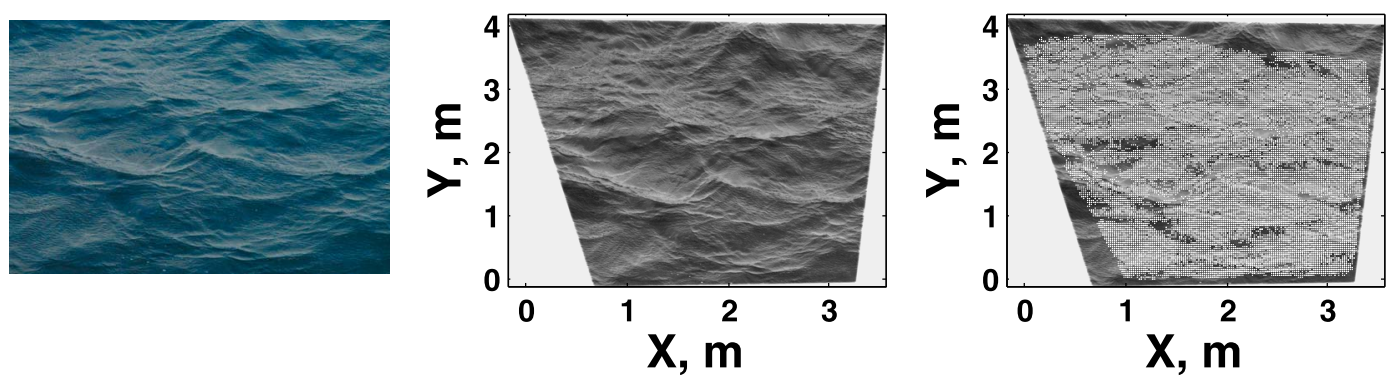

Figure 3. Example of reconstructed surface. (left) Snapshot from one camera. (middle) Orthotransformed common field of view of the two cameras. (right) Reconstructed points on the surface. 
Table 2. Standard Deviation $(\sigma$, in $\mathrm{cm})$ and Skewness $\left(\lambda_{3}\right)$ of the Distribution of Surface Elevations as Derived From Gauge and Stereo Data With and Without Detrending or Filtering

\begin{tabular}{lcc}
\hline & $\sigma($ in cm) & $\lambda_{3}$ \\
\hline & Case 1 $\left(L_{c}=1.5 \mathrm{~m}\right)$ & \\
Gauge all scales & 7.3 & -0.1 \\
Gauge filtered & 1.3 & 0.16 \\
Stereo image all scales & 3.5 & $0.17 \pm 0.9$ \\
Stereo image detrended & 1.4 & $0.13 \pm 0.3$ \\
& & \\
Gauge all scales & Case 2 $\left(L_{c}=1.5 \mathrm{~m}\right)$ & 0.1 \\
Gauge filtered & 22.3 & 0.23 \\
Stereo image all scales & 1.5 & $-0.12 \pm 1.2$ \\
Stereo image detrended & 10.3 & $0.14 \pm 0.5$ \\
& 1.5 & 0.16 \\
Gauge all scales & & 0.27 \\
Gauge filtered & Case 3 $\left(L_{c}=1.235 \mathrm{~m}\right)$ & $0.19 \pm 0.5$ \\
Stereo image all scales & 22.8 & $0.18 \pm 0.4$ \\
Stereo image detrended & 2.2 & 19.4 \\
\hline
\end{tabular}

coordinates over a frame. It clearly evidences the lacunary as well as gridded nature of the distribution of points.

[29] The aforementioned issues must be addressed and corrected when performing a systematic statistical analysis of the data set.

\section{Distribution of Sea Surface Elevations}

\subsection{Technical Issues and Their Solutions}

[30] In this section we will evaluate the distribution of elevations and compare it with both the results from the literature and concurrent wave gauge measurements. The estimation of elevations is clearly affected by issues I1 (vertical accuracy), I2 (limited range of scales) and I3 (missing mean plane) mentioned above. The last issue (I6) is less crucial since the distribution of elevation is mainly imposed by the largest waves. It limits the accuracy in the estimation of the small amplitudes distribution but has negligible impact in the estimation of the moments of the distribution. The points I1 and I2 are more problematic. We recall that the elevations on each image are given relative to their mean value over the patch. The lack of absolute height variation with respect to an overall mean plane makes it impossible to estimate the distribution of large amplitudes. However, we can estimate the distribution of small scales, by which we mean smaller than the patch size. To do this, we must address additionally the second issue (I3) pertaining to the mean tilted plane. Both I 2 and I 3 can be solved by assuming that the surface process can be decomposed in the form $\eta=\eta_{\text {detrend }}+\eta_{\text {trend }}$, where $\eta_{\text {detrend }}$ is the small-scale process and $\eta_{\text {trend }}$ is the trend due to large scales. The terms "small" and "large" are understood with respect to the size of the imaging patch. The trend will be sought in the form of a bivariate polynomial of $\operatorname{order} N$,

$$
\eta_{\text {trend }}(\boldsymbol{r})=\sum_{n=0}^{N} \sum_{j=0}^{n} a_{i, j} x^{i} y^{j-i},
$$

where $\boldsymbol{r}=(x, y)$ is the coordinates of the point and the coefficients $a_{i j}$ are found by a least-square minimization.
Removing this polynomial trend has the double effect of filtering the large scales and removing the unknown planar tilt. However, the mathematical operation of detrending is not a perfect low-frequency filtering procedure. It is characterized by a complicate transfer function that is an approximate high-pass filter. It is intuitively clear that trend removal will affect dominantly the long waves, but it also has a side effect on short-waves that is a priori unknown. In particular the spectral density and probability distribution function of the detrended elevations depends on the order of the detrending polynomial and could be different from the small-scale process resulting from a sharp high-pass filter. The choice of an at least cubic rather than linear trend is natural since long waves are not flat enough to be removed by a simple plane. On the other hand, increasing the order of the detrending polynomial improves the removal of long waves but also filters out shorter and shorter waves that are better matched by the oscillations of the polynomial inside the patch. Hence, the choice of a third-order polynomial seems to be a good compromise to get rid of wavelengths larger than the patch without affecting too much the shorter wavelengths. At this stage, we have no general argument to evaluate the artifact of detrending on the small-scale process and we must proceed empirically to find the effective cut-off frequency.

[31] A solution to determine what is the actual transfer function of a third-order trend removal is to perform a comparison with a set of reference gauge measurements. Contrarily to the stereo technique, wave gauges can follow long waves and are only limited by a high-frequency cut-off corresponding to the smallest elevation they can probe (about several millimeters). A sharp frequency filter can be applied to the time series to extract the small-scale process that can be in turn compared to the detrended process from the stereo technique. The threshold frequency is given by gravity wave dispersion relation,

$$
f_{c}=\sqrt{g /\left(2 \pi L_{c}\right)}
$$

where $L_{c}$ is the limiting wavelength that we expect to be close to half the patch size.

\subsection{Experimental Results}

[32] Sea surface elevations extracted from about 65-75 available stereo images were detrended and stacked to produce a well-resolved distribution of elevations. The rms elevation of detrended stereo data can be calculated indistinctly by either averaging the rms elevation of each individual frame or calculating the rms of the stacked distribution.

[33] On the other hand, from wave height time records and a further average over gauges we could extract the probability distribution function (p.d.f) of elevations for each case as well as the distribution of small-scale elevations resulting from high-pass filtering at $f_{c}$. The threshold frequency $f_{c}$ was adjusted to obtain the best match between the two available moments (rms and skewness) of the small-scale distribution. As expected, the cut-off wavelength is found to be about half the patch-size $\left(L_{c}=1.5\right.$ meter for case 1 and $2, L_{c}=1.235$ for case 3$)$.

[34] Table 2 shows the values of the rms and skewness coefficients for the all-scale and small-scale distributions 

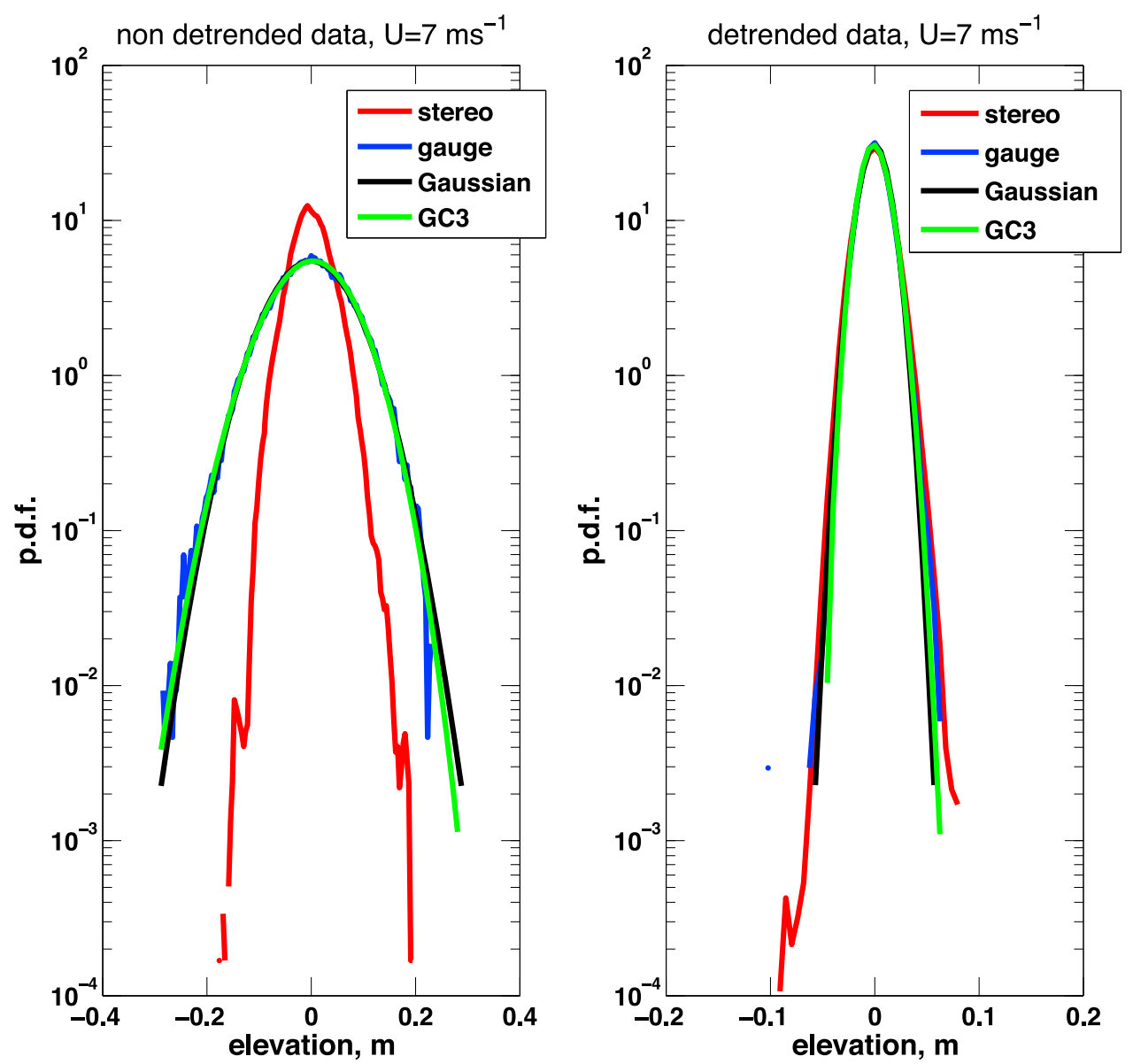

Figure 5. Comparison of the sea surface elevation distribution function obtained from stereo data and obtained from wave gauge for case 1; (left) height distribution for the original data sets; (right) corrected elevation-distribution with large scale waves information removed. The Gaussian distribution and the third-order Gram-Charlier correction (GC3) are given for reference.

from both stereo and gauge measurements, as well as the optimal threshold wavelength $L_{c}$. The small-scale rms increases slightly with wind (from about $1 \mathrm{~cm}$ to $2 \mathrm{~cm}$ ) while the skewness of elevation is found at a rather constant positive value, $\lambda_{3}=0.1-0.2$, in accordance with the values obtained with the filtered gauge. However, a large dispersion is observed on the coefficients $\lambda_{3}$ and $\lambda_{4}$ from one frame to another. This indicates that the calculation of the higher moments suffers from a lack of accuracy due to the small size of the frames and their limited number. The standard deviation of the values $\lambda_{3}$ and $\lambda_{4}$ calculated on individual frame provides a confidence interval for the estimated value. We found no reliable estimate for the kurtosis coefficient $\lambda_{4}$ that is subject to such dispersion that its average value is meaningless.

[35] Figures 5-7 show the elevated p.d.f from the wave gauge as well as from the stereo image data. The distribution is plotted in semi-logarithmic representation in order to evidence the center as well as the tail. The p.d.f derived from wave gauge suffers from strong oscillations in their tail because of the limited length of the record and the limited number of gauges (2 to 6). This has a strong negative impact on the tail of the p.d.f of the filtered gauge in cases 2 and 3 and prevents from an accurate estimation of the higher moments. In each case the Gaussian distribution with same variance as well as the third-order Gram-Charlier correction (that is including skewness $\lambda_{3}$ ) is given for reference. As seen, the departure from Gaussian increases with wind speed and is more pronounced for the distribution of small-scale elevations. Note that the lowest-order Gram-Charlier correction is not sufficient to reproduce the broadening of the distribution of small-scale elevations observed at large values. The p.d.f corresponding to the unfiltered data sets (raw gauge and non detrended stereo data) are dramatically different as they do not address the same scales. However, after filtering of the wave gauge and detrending of the stereo data, a good agreement between filtered gauge and detrended stereo data is observed, except in the tail of the distribution where the accuracy of the gauge p.d.f is questionable.

[36] Hence, the probability density function of smallscales (that is smaller than about 1.5 meter) can be consistently recovered from stereo data after a detrending operation. We are not able, however, to evaluate the accuracy of 

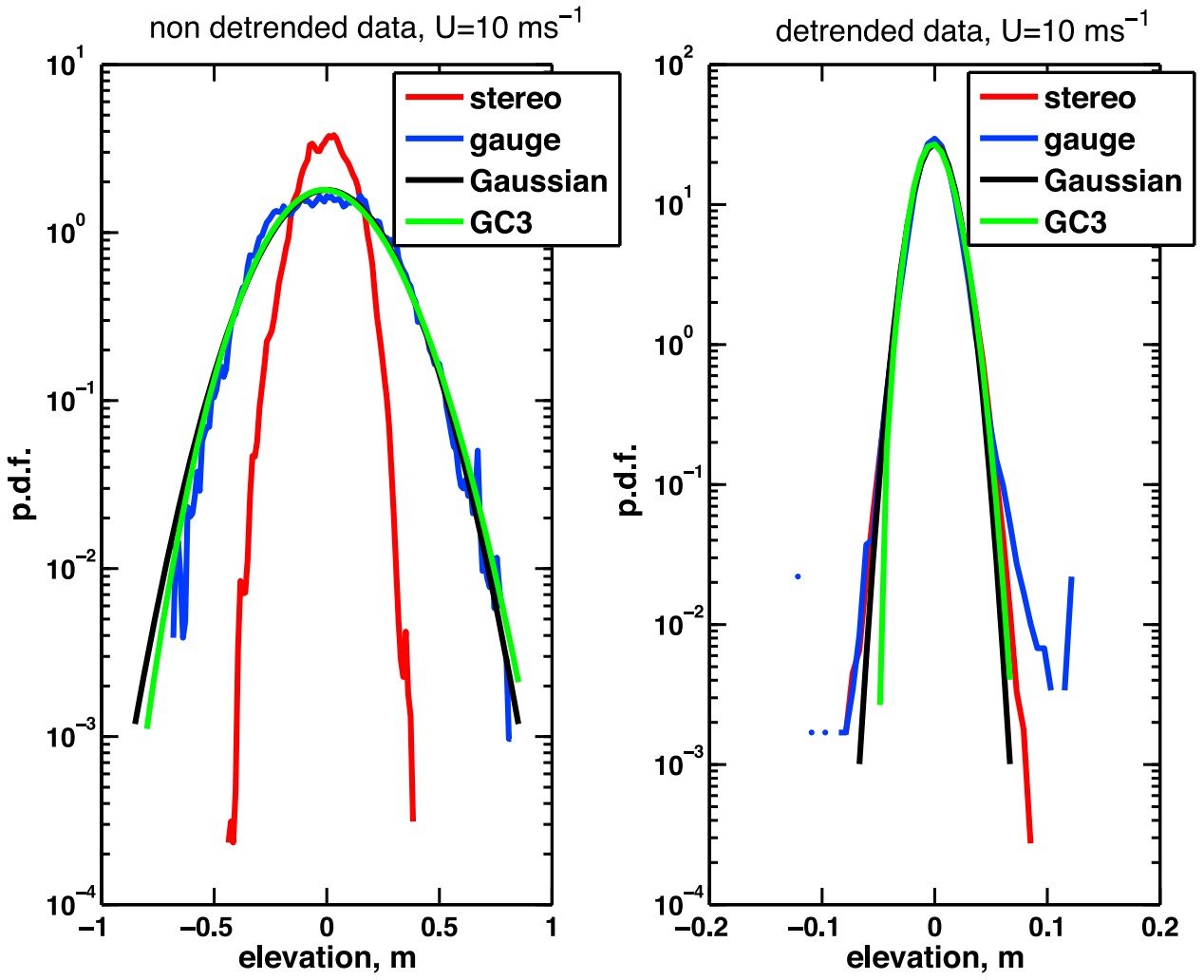

Figure 6. Same as Figure 5 for case 2.
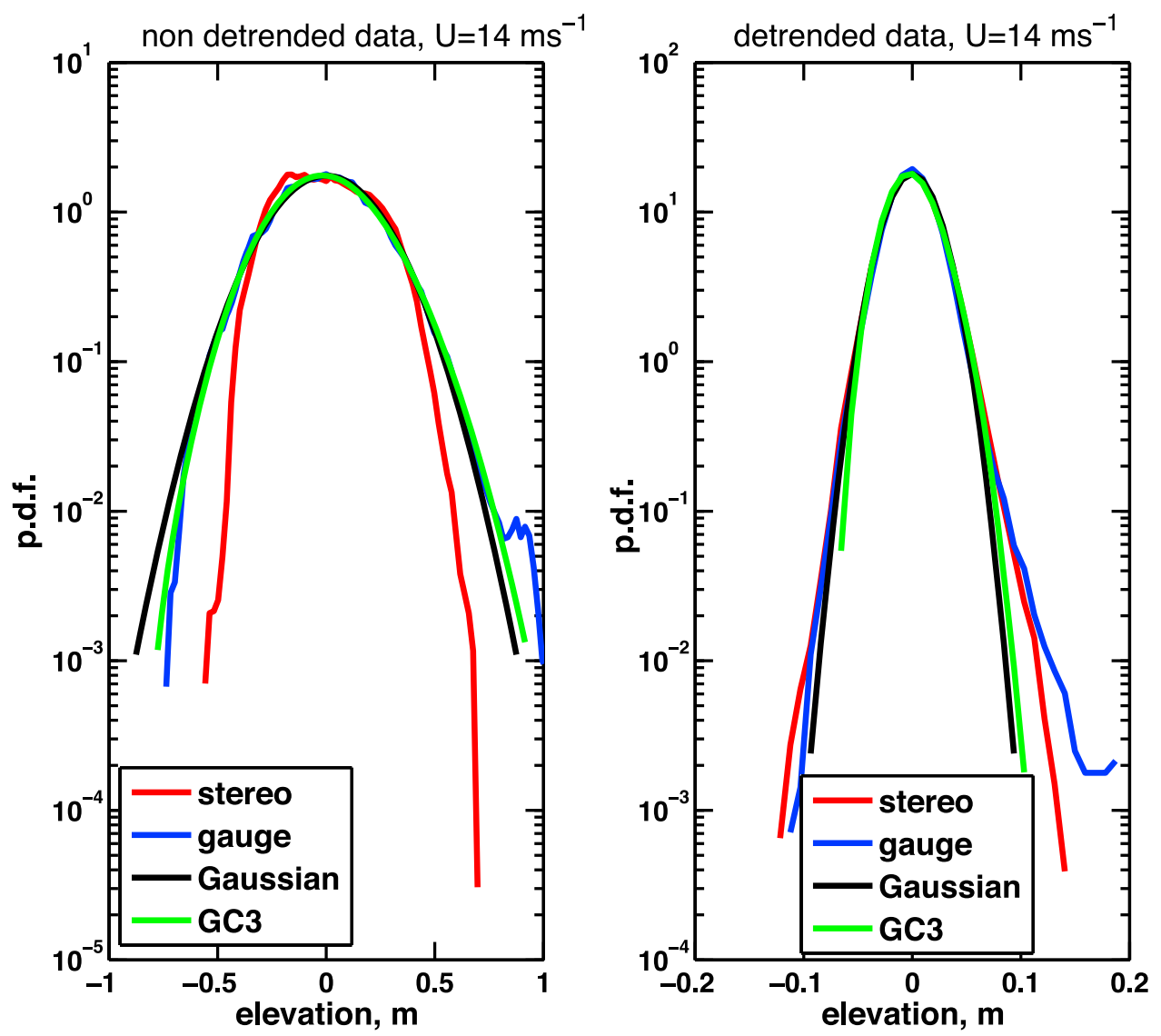

Figure 7. Same as Figure 5 for case 3. 


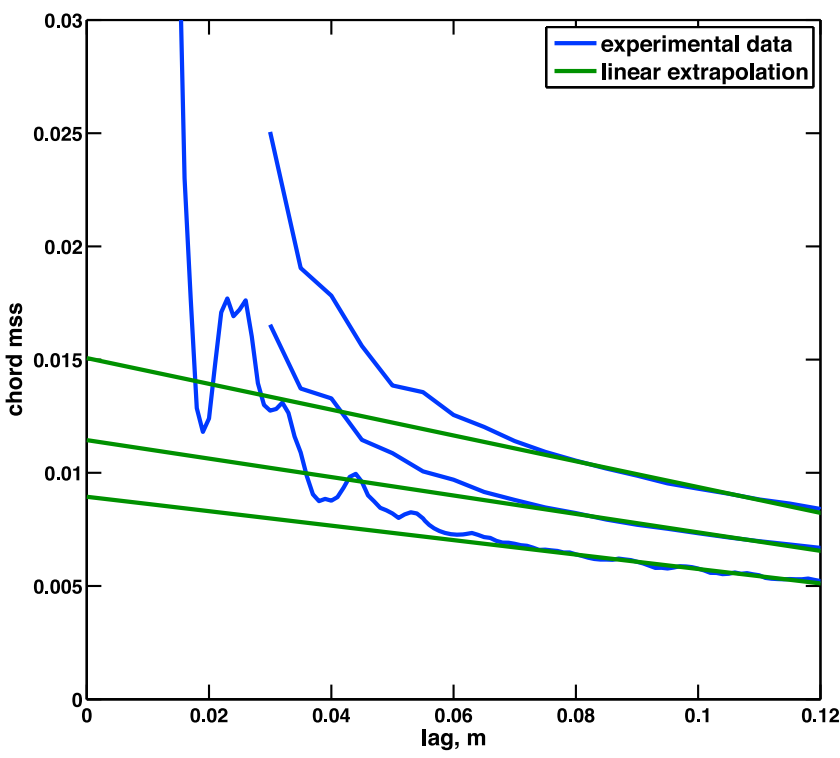

Figure 8. Estimation of the small-scale mss by extrapolation of the chord mss at small but finite lags. Bottom curve: case 1, middle curve: case 2 and upper curve: case 3.

this retrieval procedure in view of the insufficient quality of the reference gauge measurements.

\section{Distribution of Sea Surface Slopes}

\subsection{Technical Issues and Their Solutions}

[37] The estimation of slope suffers from the limitations I1-I6 for the following reasons:

[38] Il (horizontal accuracy) - The slopes of a discrete surface are in general computed via finite differences over nodes:

$$
\eta_{x}=\frac{\eta\left(x_{n+1}, y_{n}\right)-\eta\left(x_{n}, y_{n}\right)}{x_{n+1}-x_{n}}, \eta_{y}=\frac{\eta\left(x_{n}, y_{n+1}\right)-\eta\left(x_{n}, y_{n}\right)}{y_{n+1}-y_{n}},
$$

This simple technique is sufficient whenever the surface is well resolved, that is, if it is band-limited with a sampling meeting the conditions of Shannon theorem. Now although the distance between nearest neighbors is about $1 \mathrm{~cm}$ while, as it is well known, millimeter features still contribute significantly to the total slope.

[39] I1 (vertical accuracy)-As mentioned, the vertical accuracy of retrieved elevations is mainly determined by parallax effects. For present experimental set-up and conditions the estimated error is a of few millimeters. However, additional errors are possible due to incorrect geometrical treatment of sporadic unsuspected features on the sea surface, e.g., separate foam bubbles and flying drops. These errors can be seen as unrealistic spikes on the reconstructed sea surface. This induces a noticeable bias in the estimation of slope since the difference of elevations at neighbor points can be of the order of magnitude of the accuracy.

[40] I2-I3- The limited range of scale a priori eliminates the contribution of small- $(<1 \mathrm{~cm})$ and large- $(>3 \mathrm{~m})$ slopes. The slopes of the missing large scales can in principle be recovered by averaging the mean facet of every image. However, the poor estimation of the mean tilted plane and the small number of images $(<100)$ introduces a large bias in this calculation.

[41] I4-I5-I6-Irregular sampling and gaps make the utilization of a finite difference scheme (24) hazardous since artificially small slopes can be obtained by filling the gaps between distant neighbor points. Interpolation must be avoided because it misses the contribution of sub-resolution scales and creates artificially flat surfaces between the gaps. At last, the gridding effect mentioned earlier produces a strong artifact on the slope estimation with a finite-difference scheme. It produces different results according to whether the reference axis $(\mathrm{x}, \mathrm{y})$ are chosen parallel to the elementary cell or along a diagonal.

[42] The issue pertaining to missing large scales (I2) and mean tilted plane (I3) can again be addressed by the detrending procedure. The artifact of lacunary, irregular and gridded sampling (I4-I5-I6) can be avoided by evaluating the slope from the omnidirectional structure function (equation (9)). The limitation due to the limited small-scale resolution can be overcome by inferring the limit of the ratio $S_{2}(r) / r^{2}$ and $S_{4} / S_{2}^{2}$ from a simple linear extrapolating to the origin.

\subsection{Experimental Results}

[43] The structure functions at finite lag have been computed from an ensemble average over frames and doublets at a prescribed distance $r \pm \Delta r$, with a tolerance $\Delta r$ set to $0.5 \mathrm{~cm}$ that corresponds approximately to the expected resolution. To estimate the error $\Delta S_{2}$ induced by this tolerance interval we can assume a quadratic form $S_{2}(r) \sim$ $\frac{1}{2}$ mss $r^{2}$ that implies a relative error $\Delta S_{2} / S_{2} \sim 2 \Delta r / r$, that is less than $10 \%$ for lags greater than $5 \mathrm{~cm}$. Figures 8 and 9 show the estimation of these parameters from the structure function $S_{2}$ and $S_{4}$ by linear extrapolation of relations (9) and (11) for the all wind cases. A good linear approximation of the chord mss $\left(S_{2} / r^{2}\right)$ can be found for lags larger than about $7 \mathrm{~cm}$ and the extrapolated value is little sensitive to the precise value of this threshold. The quality of the linear

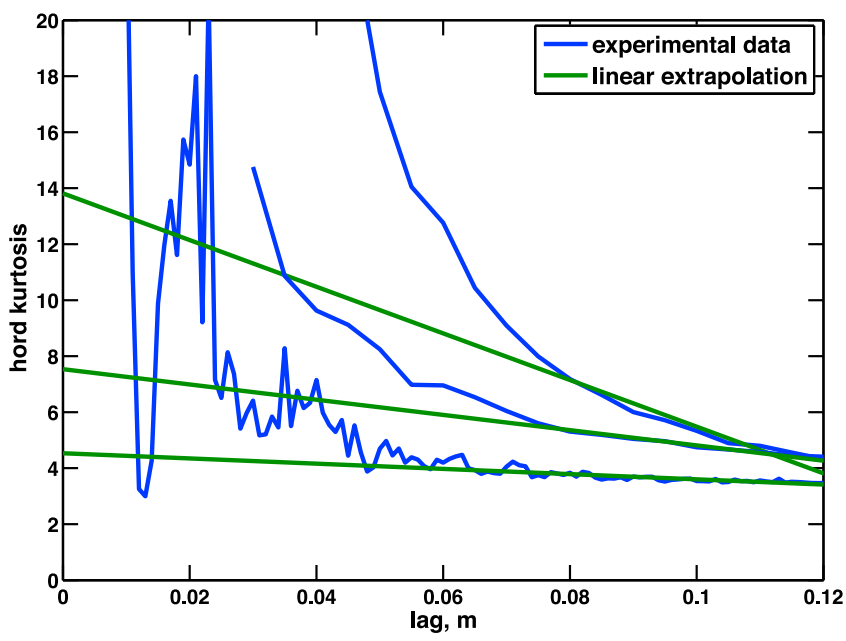

Figure 9. Estimation of the kurtosis of small-scale slopes by extrapolation of the chord kurtosis at small but finite lags. Bottom curve: case1, top curve: case 2 and middle curve: case 3 . 
Table 3. Estimation of the mss and the Omnidirectional Kurtosis of Small-Scale Slopes ${ }^{\mathrm{a}}$

\begin{tabular}{lccc}
\hline & \multicolumn{3}{c}{ Wind } \\
\cline { 2 - 4 } & 7 & 10 & 14 \\
\hline $1000 \times$ mss clean CM (all scales) & 39 & 54 & 74 \\
$1000 \times \Delta_{1}$ mss (scales $\left.<30 \mathrm{~cm}\right)$ & 18 & 29 & 36 \\
$1000 \times \Delta_{2}$ mss $($ scales $<1-2 \mathrm{~m})$ & 23 & 34 & 50 \\
$1000 \times$ mss detrended stereo & 18 & 24 & 31 \\
$\quad($ scales $<1.5$ m) & 4.5 & & 7.7 \\
$\mathrm{~K}_{\text {omni detrended stereo }}$ & & - & \\
\hline
\end{tabular}

${ }^{\mathrm{a}}$ The quality of the regression was too poor in the second case to provide an estimation of the kurtosis.

regression is much poorer in the case of the chord kurtosis $\left(S_{4} / S_{2}^{2}\right)$ and the range of values over which it should be applied is not well defined. This induces a larger margin of error in the estimation of the omnidirectional kurtosis of slopes, $\mathrm{K}_{\mathrm{omni}}$. We were, however, not able to quantify this error. Note that no satisfying linear approximation could be found in case 2 .

[44] The reference comparison for the estimation of mss is the set of values reported by Cox and Munk [1954], henceforth referred to as CM. Due to the difference of sea conditions between the places where the experiments were run (close versus open sea, short versus long fetch, etc.) it is not meaningful to compare the exact values of mss even at same wind speeds. However, the order of magnitude and evolution with wind should be comparable. We recall that $\mathrm{CM}$ measured the slopes at the sea surface in two different conditions referred to as "clean" and "slick" case, respectively. The mss obtained in clean sea conditions involves all wave scales while the mss measured on an oil-covered slick sea surface pertains to scales larger than about $30 \mathrm{~cm}$ (smaller scales being damped by the increased viscosity). As mentioned before, the mss estimated from stereo data addresses the small-scale roughness only, that is waves smaller than the patch size. Hence, it should be definitely smaller than the clean mss but larger or comparable to the increase of mss from "slick" to "clean", which corresponds roughly to the contribution of scales smaller than $30 \mathrm{~cm}$ :

$$
\Delta_{1} \mathrm{mss}=\text { clean mss }- \text { slick mss }
$$

[45] The mss of the detrended surface can also be meaningfully compared with the airborne experiment described in Vandemark et al. [2004], referred to in the following as VDM. The slope vector of one-meter chords at the surface along the track, say $\boldsymbol{s}=s_{x} \hat{\boldsymbol{r}} x+s_{y} \hat{\boldsymbol{y}}$, was measured by means of a system of vertical lasers mounted one meter apart. The 'large mss' is the variance of the slope vector averaged over all directions:

$$
\text { large mss }=\left\langle\left\|s^{2}\right\|\right\rangle=\left\langle s_{x}^{2}\right\rangle+\left\langle s_{y}^{2}\right\rangle
$$

[46] This quantity can be interpreted as the mss of scales larger than 1-2 meter, that is precisely the scales that are missed in the stereo analysis. Now we can consider the increment of mss from small to intermediate scale, namely

$$
\Delta_{2} \mathrm{mss}=\text { clean mss }- \text { large } \mathrm{mss}
$$

where the clean mss is, again, the total slope measured by $\mathrm{CM}$. These increments of slopes should be comparable to the estimated slope on detrended stereo data. Table 3 reports the estimated mss in the three wind cases for the stereo data as well as the aforementioned mss and increments of mss. Even though the order of magnitude is found correct, the mss obtained from the stereo data seem to be slightly underestimated in view of the previous considerations, especially at larger winds. The discrepancy can be due to different factors: the difference in the sea states of the various set of data that are not truly comparable, the lack of accuracy in the slope estimation with the current procedure or the inability of the stereo imaging technique to recover the large slopes at the sharper crests of waves. The clarification of this point requires further investigation. As to the values of kurtosis reported in Table 3 they can only be commented qualitatively in view of their large uncertainty. All that can be asserted is that the kurtosis increases significantly with wind and is much higher than the value $\mathrm{K}_{\mathrm{omni}} \sim 3.15$ following from (12) and CM measurements. This supports the wellknown fact [e.g., Caulliez and Guérin, 2012] that the slopes of short waves exhibit much higher kurtosis than long waves.

\section{Distribution of Chords}

\subsection{Technical Issues}

[47] In contradistinction to surface slopes, structure functions at intermediate length can be measured accurately as they do not suffer from the same limitations. This is because the slope of larger chords are less affected by both the limited (horizontal and vertical) resolution and the gridding effect that makes the estimation of slopes problematic. However, they still suffer from the limitations I1 and I2 owing to the large scales. Again, the data have been detrended to eliminate the large-scale contribution.

\subsection{Comparison With Gauge Wire}

[48] The wire gauge consists of six strings located at the nodes and center of a pentagon of diameter $50 \mathrm{~cm}$. By evaluating the instantaneous difference of elevations between the central gauge and the other five gauges, one can estimate the statistics of chords of length $25 \mathrm{~cm}$ in five regularly spaced directions with respect to wind. To compare this chord distribution with stereo data, the large scales have been filtered by removing the frequencies lower than the threshold frequency $f=\sqrt{g /(2 \pi L)}=1 \mathrm{~Hz}$, where $L=1.5 \mathrm{~m}$ is the size of the patch. The omnidirectional chord mss is obtained by angular average of the directional chord mss. Due to the small number of gauges, it is preferable to estimate the average of the chord kurtosis in every direction $\left(\lambda_{4}^{\text {dir.av }}\right)$ rather than the omnidirectional kurtosis $\left(\lambda_{4}^{\text {omni }}\right)$. The mss and kurtosis of chord in each direction were computed from stereo data in angular sectors of 72 degrees with a tolerance of $\pm 5 \mathrm{~mm}$ on the radius $\mathrm{r}=25 \mathrm{~cm}$. In addition to the simultaneous wave gauges measurements, the chord mss can be, again, meaningfully compared with the optical measurements of CM and VDM. As a matter of fact, the $25 \mathrm{~cm}$ chord mss is roughly equivalent to the mss of the smoothed surface (with no components smaller than about $25 \mathrm{~cm}$ wavelength) while the detrending process removes the scales larger than about half the patch size (see section 5). Hence the $25 \mathrm{~cm}$ chord mss of detrended data is comparable to the increment of mss between $30 \mathrm{~cm}$ and 1-2 meter, that is 
Table 4. Mean Square Slope and Directionally Averaged Kurtosis of $25 \mathrm{~cm}$ Chords Derived From Filtered Wave Gauges (wg) and Detrended Stereo Images (si)

\begin{tabular}{lccc}
\hline & \multicolumn{3}{c}{ Wind } \\
\cline { 2 - 4 } & 7 & 10 & 14 \\
\hline $1000 \times$ mss wg & 4.6 & 5.1 & 5.2 \\
$1000 \times$ mss si & 3.5 & 4.3 & 5.6 \\
$1000 \times \Delta_{3}$ mss & 5 & 5 & 14 \\
$\lambda_{4}^{\text {dir.av }}$ wg & 3.3 & 3.4 & 3.9 \\
$\lambda_{4}^{\text {dir.av }} \mathrm{si}$ & 3.3 & 3.3 & 3.7 \\
\hline
\end{tabular}

precisely to the difference between the aforementioned slick and large mss:

$$
\Delta_{3} \mathrm{mss}=\text { mss slick }- \text { mss large }=\Delta_{2} \mathrm{mss}-\Delta_{1} \mathrm{mss}
$$

Table 4 reports the estimation of the chord mss and kurtosis for detrended stereo data and filtered wave gauges as well as the incremental slope $\Delta_{3}$ mss for the three wind speeds. The chord mss and kurtosis estimated from the stereo data are found consistent with both the wave gauges and the slope increments, except for the strongest wind where the chord mss $\Delta_{3}$ mss estimated from airborne measurements is larger by a factor 3 . This is linked to the discrepancy already observed in Table 3 for the small scale mss.

\section{Calculation and Consistency of the Spectrum}

\subsection{Direct Fourier Technique}

[49] As mentioned previously, stereo imaging gives access to an intermediate range of scale with a low-wave-number cut-off imposed by the field of view and a high-wavenumber cut-off due to the limited resolution. After removal of the large-scale trend this is, however, informative as it permits an estimation of the surface elevation density spectrum in the given range of wave numbers. This has been detailed in Kosnik and Dulov [2011] and shown consistent with the low wavenumber part of the spectrum evaluated by means of wave gauges.

[50] We briefly recall the procedure that has been used in Kosnik and Dulov [2011] to estimate the elevation spectrum from stereo data by means of a direct Fourier technique, a method that we henceforth refer to as "DFT". Reconstructed surfaces are first detrended by removing the mean bicubic surface in order to exclude large waves. Patches of the surface are selected (about 10-15 per frame) and their individual spectra calculated using Fast Fourier Transform method with Hann windowing. The side of every small rectangular area is about $0.5-2$ meter depending on the quality of the reconstructed surface, i.e. the proportion of gaps. The spectra obtained for the different pieces of image are interpolated on regular wave number bins and averaged over the frame. A further ensemble average is performed over surface samples (about 70). The omnidirectional spectrum is obtained by angular integration of the two-dimensional spectrum. Such a technique limits the estimation of the spectrum to the wave number range $30 \mathrm{rad} \mathrm{m}^{-1}<k<60 \mathrm{rad} \mathrm{m}^{-1}$. Here, in this paper, we do not take into account the brightness-based higher-frequency spectrum extension method described in Kosnik and Dulov [2011].

\subsection{Frequency and Wave-Number Spectrum}

[51] Wave gauge data, unlike stereo data, are distributed in time but not in space and a comparison requires the conversion of a frequency spectrum into a wave-number spectrum. The dispersion relation of deep water gravity waves $\left(k=4 \pi^{2} f^{2} / g, S(k)=S(f) \sqrt{g / k} /(4 \pi)\right)$ was used for this procedure. The frequency spectra have been processed over 20 minute records. However, to smooth out the oscillations, the spectrum has been calculated over shorter overlapping intervals and averaged. In field conditions, wave gauge measurements provide correct frequency spectrum up to about $f \simeq 1.5 \mathrm{~Hz}$, the higher frequencies being corrupted by the orbital velocity of the dominant wave [Banner, 1990].

\subsection{Autocorrelation Function Technique}

[52] Stereo images can also provide the autocorrelation function of sea surface elevation $(\eta)$ :

$$
\rho_{2}(\boldsymbol{r})=\left\langle\eta\left(\boldsymbol{r}_{1}\right) \eta\left(\boldsymbol{r}_{2}\right)\right\rangle-\left\langle\eta\left(\boldsymbol{r}_{1}\right)\right\rangle\left\langle\eta\left(\boldsymbol{r}_{2}\right)\right\rangle
$$

where as usual the brackets $\langle\cdot\rangle$ denote the ensemble average over sample surfaces and $\left(\boldsymbol{r}_{\mathbf{1}}, \boldsymbol{r}_{\mathbf{2}}\right)$ is any pair of point at a prescribed distance $\boldsymbol{r}=\boldsymbol{r}_{\mathbf{1}}-\boldsymbol{r}_{\mathbf{1}}$. Note that we thus build a stationary autocorrelation function, that is we assume the roughness process to be homogeneous in space.

[53] The spatial extent of the reconstructed topography and its effective resolution gives access to the spectral range $5 \mathrm{rad} \mathrm{m}^{-1}<k<200 \mathrm{rad} \mathrm{m}^{-1}$. While the long wave spectrum is not accessible by this technique the structure of short waves is better described than in the case of wave gauges.

[54] However, an accurate estimation of the autocorrelation and higher-order structure functions from stereo images requires much caution as unphysical artifacts caused by interpolation and the presence of gaps must be avoided. To evaluate the function as given in (29) we consider pairs of points $\boldsymbol{r}_{\mathbf{1}}, \boldsymbol{r}_{\mathbf{2}}$ obtained from linear interpolation of the irregular grid on the distance with radius between $r$ and $r+\Delta r$. The tolerance $\Delta r$ is set to $1 \mathrm{~cm}$, which is approximately the resolution of the experimental data. This makes it possible to evaluate the correlation function (as well as structure function of any order) without interpolation over large distances. In addition the obtained function is regularly sampled and thus suitable for the "traditional" discrete analysis techniques, e.g., the Fast Fourier Transform.

\subsection{Consistency Between the Different Approaches}

[55] A correct calculation of the autocorrelation function is a prerequisite for the estimation of higher-order structure functions that necessitate increasing accuracy in the estimation of the difference of elevations. It is also a consistency test for the calculation of the directional density spectrum with DFT. To render the comparison possible the omnidirectional spectrum has been calculated by angular average of the directional density spectrum. Figure 10 shows the omnidirectional spectra obtained by the three techniques, namely DFT (black line), autocorrelation function technique (red line) and wave gauge wavenumber spectrum (thin black line). A very good agreement is found between the DFT and the autocorrelation function method in their common range 

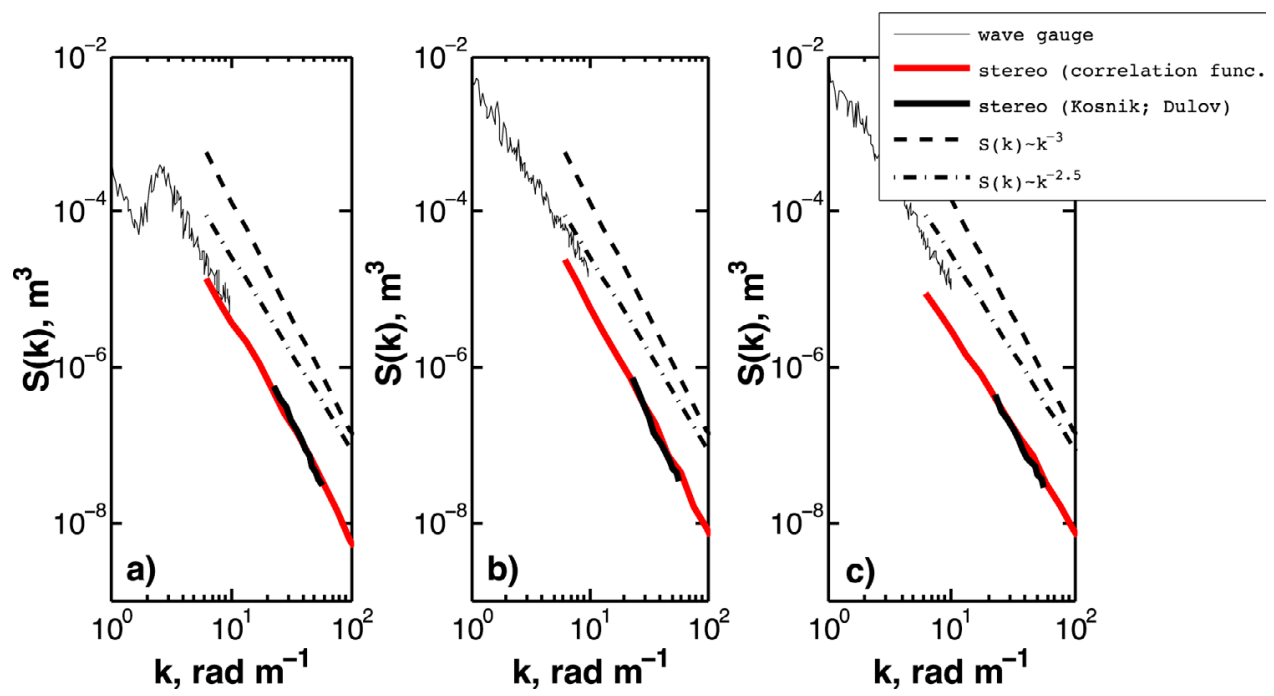

Figure 10. Comparison of omnidirectional wave number spectra obtained at different wind speeds from wave gauge and stereo data with either Fourier processing [Kosnik and Dulov, 2011] or autocorrelation function method. (a) Case 1; (b) case 2; (c) case 3.

of wave numbers. However, spectrum based on the autocorrelation technique addresses a larger range of wavenumbers. The reason is that the image needs not be split into small pieces as with DFT. Comparison with the wave gauge frequency spectrum is satisfactory except for the strongest wind case. The causes of discrepancy between the frequency wave gauge spectrum and wavenumber spectrum from stereo data can be different. Classical linear dispersion relation that was used for spectra comparison, may be violated due to surface currents, orbital velocities of the longer waves, wind drift and the increasing influence of foam and breaking at high wind speeds.

[56] Another consistency test consists in recalculating the mss from the integration of the omnidirectional spectrum:

$$
\text { mss stereo }=\int_{k_{1}}^{k_{2}} k^{2} S(k) d k,
$$

where $k_{1}=6 \mathrm{rad} / \mathrm{m}$ and $k_{2}=100 \mathrm{rad} / \mathrm{m}$ are the limiting available wave numbers. Table 5 gives the estimates of mss from (30) and from the linear extrapolation of experimental structure function (9) (same as in Table 3). The values are found in good agreement. Note that the typical $k^{-3}$ fall off that is observed for the spectrum cannot be assumed valid over arbitrary large frequencies as this would imply a blow up of the mss. There is certainly a cut-off value in this spectral power-law regime that, however, is not visible in the accessible range of frequencies.

[57] This analysis has assessed the possibility to estimate the second-order structure function $\left(S_{2}\right)$ from stereo data with good accuracy in the range $0.05 \mathrm{~m}<r<0.9 \mathrm{~m}$. This validation step was necessary for evaluation of the level of experimental errors before turning to the study of the skewness function.

\section{Skewness Function}

[58] In view of the multi-scale and oscillating nature of the sea surface, the structure functions of arbitrary orders have also an oscillating behavior. It was observed in a recent experimental study [Caulliez and Guérin, 2012] that the period of these oscillations is of the order of the dominant wave. Hence positive parametrizations such as (18) and (19) are only expected to hold at small distances compared to the peak wave. In that case the current set of data makes it possible to check the validity of these parametrizations. In view of the limited range of available scales the structure function of the total elevation is not attainable beyond very small lags. However, we can compute the structure function associated to the smallscale process, say $\eta_{s}$, obtained after detrending of the large scale, say $\eta_{L}$. The statistical characterization of the small-scale process is relevant in the context of the TwoScale or composite scattering model, which writes the radar cross section as the superposition of two terms. The first term is the contribution of large scales through a Geometrical Optics mechanism. The second term is the field scattered by small rippled on tilted facets. Limiting the skewness function to small-scales is further justified by the fact that the large-scale skewness function is in fact not needed at small lags. This can be understood in a Two-Scale picture where the sea surface is considered to be the superposition of one large- and small-scale (centered) process, $\eta=\eta_{L}+\eta_{s}$, which we assume to be statistically independent although it is not hydrodynamically true. Assuming minimum scale $\sim L$ for the large-scale process we may approximate $\eta_{L}(\boldsymbol{r}) \simeq \eta_{L}(0)+\nabla \eta_{L}(0) \cdot \boldsymbol{r}$ in the

Table 5. Estimation of the mss With Expression (30) and Linear Interpolation of Experimental Function (9)

\begin{tabular}{lccc}
\hline & \multicolumn{3}{c}{ Wind } \\
\cline { 2 - 4 } & 7 & 10 & 14 \\
\hline mss, autocorrelation function & 0.018 & 0.026 & 0.028 \\
mss, extrapolation of equation (9) & 0.018 & 0.024 & 0.031 \\
\hline
\end{tabular}



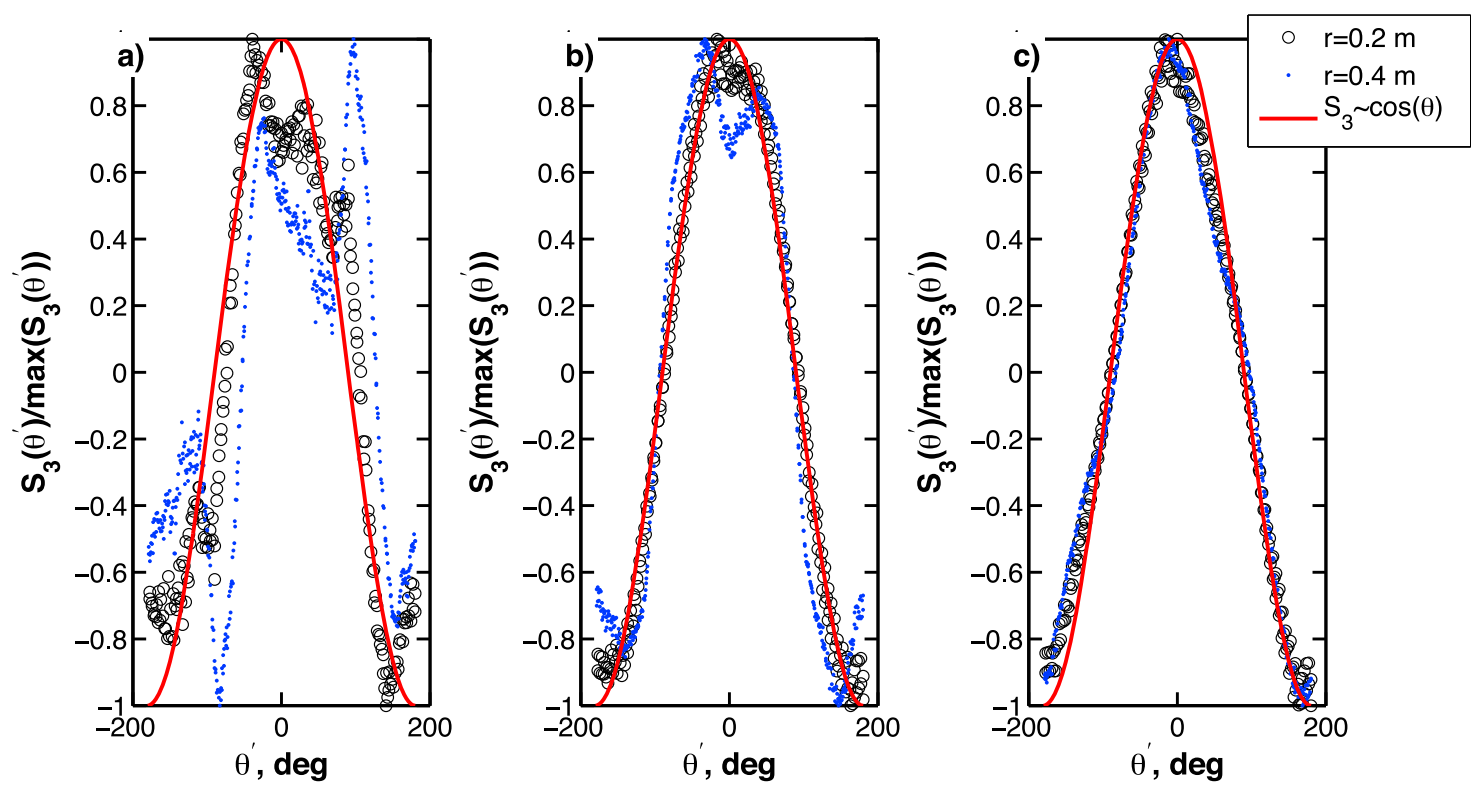

Figure 11. Directional behavior of the skewness function for different constant lags (here $\theta^{\prime}=\theta-180^{\circ}$ ).

(a) Experimental case 1; (b) experimental case 2; (c) experimental case 3.

range $r \ll L$ and a simple calculation yields the following approximation for the resulting skewness function in this range of lags:

$$
\left.S_{3}(\boldsymbol{r})\right|_{\text {all scales }} \simeq\left\langle\left(\boldsymbol{r} \cdot \nabla \eta_{L}\right)^{3}\right\rangle+\left.S_{3}(\boldsymbol{r})\right|_{\text {small scales }}
$$

[59] Now the skewness coefficients of slopes are known to be much smaller for long waves than for short wave (in view of the values derived by Cox and Munk [1954] in the "clean" and "slick" case). Since the mss is well distributed across the scales (so that the small- and large scale mss are of the same order of magnitude), this means that $\left|\left\langle\left(\nabla \eta_{L}\right)^{3}\right\rangle\right| \ll\left|\left\langle\left(\nabla \eta_{s}\right)^{3}\right\rangle\right|$ and so the first term on the righthand side of (31) can be discarded. This shows that in the end only the small-scale skewness function is needed.

[60] To compute the skewness function associated to the small-scale process, the data have been detrended and interpolated on a regular grid with a third order polynomial. Again, to avoid "filling" the gaps in the reconstructed surface with artificially smooth patches, we limited the interpolation to points no further apart than $0.8 \mathrm{~cm}$. The directional dependence of the skewness function is illustrated in Figure 11 for different lags in the three wind cases. At small lags a $\cos \theta$ azimuthal dependence is clearly identified. This is consistent with the parametrization (18) in Mouche et al. [2007]. However, for large lags and small winds directional properties are different. This can be easily understood since the parametrization based on the limiting behavior of the skewness function at small lags is no longer relevant when the skewness function starts oscillating, that is for lags comparable to the dominant wave (see the discussion above). As to the dependence upon lag, our analysis shows that the relevant parametrization is (18) together with an exponential cut-off:

$$
S_{3}(\boldsymbol{r})=\frac{1}{3} r^{3} \sigma_{x}^{3} C_{30} \cos (\theta) \exp \left\{-\left(\frac{r}{r_{0}}\right)\right\}
$$

The validity of the constant factor $\frac{1}{3} \sigma_{x}^{3} C_{30}$ can be checked by evaluating the renormalized quantity $\alpha=S_{3} /\left(r^{3} \cos (\theta)\right)$ in the limit $r \rightarrow 0$. As discussed in section 5 it is not possible to determine accurately the structure function at short lags because of the limited resolution. However, the value of $\alpha$ can be extrapolated from larger lags. Figure 12 shows the renormalized skewness function $S_{3}(r) / r^{3}$ in semi-log scale for a given fixed direction $(\theta=\pi$, corresponding to the downwind direction). The linear fit starting at $r \sim 15 \mathrm{~cm}$ confirms the exponential form of the cut-off. At distance shorter than about $15 \mathrm{~cm}$, the

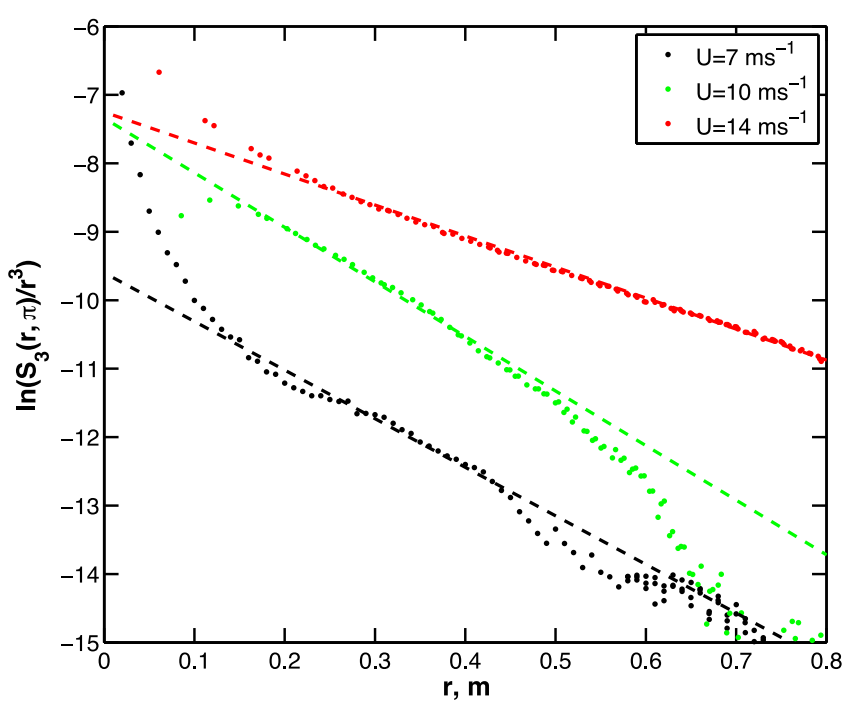

Figure 12. Natural logarithm of the rescaled skewness function $S_{3} / r^{3}$ in downwind direction for different wind speeds. Here the linear fit is performed for the lag range $r=[0.2,0.35] \mathrm{m}$ (case 1), $r=[0.2,0.4] \mathrm{m}$ (case 2), and $r=[0.2,0.8] \mathrm{m}$ (case 3$)$. 
Table 6. Amplitude Factor $\alpha=\frac{1}{3} \sigma_{x}^{3} C_{03}$ in the Skewness Function According to Cox and Munk Experimental Values (Second Column) and Derived From Stereo Data (Third Column) ${ }^{\mathrm{a}}$

\begin{tabular}{cccc}
\hline $\begin{array}{c}\text { Wind Speed } \\
\left(m s^{-1}\right)\end{array}$ & $\begin{array}{c}10^{4} \times \alpha, \\
\mathrm{CM}\end{array}$ & $\begin{array}{c}10^{4} \times \alpha, \\
\text { Stereo Data }\end{array}$ & $\begin{array}{c}r_{0}(m), \\
\text { Stereo Data }\end{array}$ \\
\hline 7 & -0.2 & -0.6 & 0.14 \\
10 & -5.4 & -6.5 & 0.12 \\
14 & -13 & -7.4 & 0.22 \\
\hline
\end{tabular}

${ }^{\mathrm{a}}$ Exponential cut-off parameter $r_{0}$ inferred from stereo data (fourth column).

renormalized function $S_{3}(r) / r^{3}$ exhibits an unphysical blow-up due to the lack of accuracy in the determination of $S_{3}$. The same was observed for the kurtosis function in section 5. The constant coefficient of the fitting lines coincides with the coefficient $\alpha$ while their slopes provide the cut-off distance $r_{0}$. The obtained numerical values are reported in Table 6 . The coefficient $\alpha$ is compared and found consistent with the theoretical factor $\frac{1}{3} \sigma_{x}^{3} C_{30}$ according to the experimental value of Cox and Munk. The cut-off distance $r_{0}=15-20 \mathrm{~cm}$ is found quite independent of wind speed.

[61] Figure 13 shows the overall quality of the presented parametric model (32) for the skewness function (shown here as a function of lag in downwind direction) and a comparison with earlier parametrizations (18) and (19) (just for the case 3, where experimental conditions are comparable). The agreement with the experimental data is excellent at lags in the range of $0.2 \mathrm{~m}<r<0.4 \mathrm{~m}$ for all wind cases and acceptable at smaller lags, where the accurate determination of the skewness function is problematic. Skewness function at large lags, where $r>$ $0.4 \mathrm{~m}$, can be described by the model (32) only for the high wind conditions. As it was discussed earlier, this can be explained by inapplicability of selected model approach for the big-scale processes close to the dominant wavelength.

\section{Conclusion}

[62] In the present paper we have proposed an extended analysis of the statistical properties of experimental sea surface elevations obtained from stereo imaging technique. This analysis has shown that reconstruction of the topography based on stereo method is an efficient way to derive non-trivial statistical properties of surface short- and intermediate-waves (say from $1 \mathrm{~cm}$ to $1 \mathrm{~m}$ ).

[63] Most technical issues, typical for stereo data sets, such as the limited range of scales, the lacunarity of data or the irregular sampling can be partially overcome by appropriate processing of the available points. The proposed technique also allows one to avoid linear interpolation that dramatically corrupts properties of retrieved surfaces. The processing technique imposes that the field of elevation is polynomially detrended, which has the effect of filtering out the large scales. Hence the statistical analysis can only address the small-scale components of the sea surface. The precise cut-off wavelength, which we know is approximatively half the patch size, can be obtained by applying a high-pass frequency filter on the reference gauge time records. The results obtained for the detrended images have been shown to be consistent, at least in order of magnitude, with the corresponding gauge measurements as well as other experimental measurements available in the literature. The calculation of the structure functions provides a powerful tool to investigate spectral and statistical properties of the field of elevations. Experimental estimation of the thirdorder structure function, namely the skewness function, is one of the most important and original results of this paper. This function was up to now unavailable in field conditions and its knowledge was limited to theoretical considerations. We have derived an experimental parametrization that
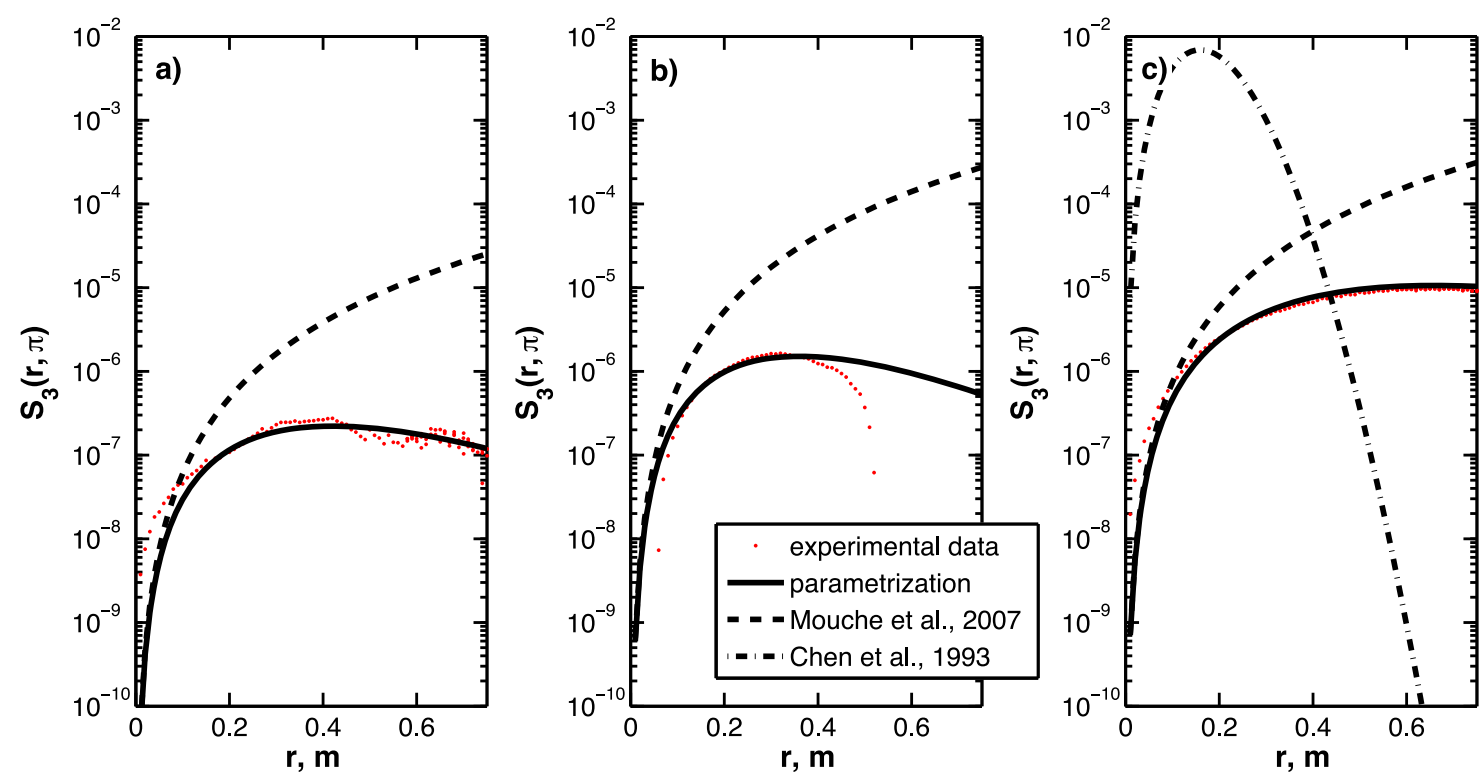

Figure 13. Comparison of experimental skewness function with existing theoretical parametrizations. (a) Experimental case 1; (b) experimental case 2; (c) experimental case 3. 
confirms a $\cos \theta$ directional behavior and an exponential cutoff form (especially for pure wind-wave sea-state).

[64] Due to the lack of precise reference measurements for the small-scale wave field, we could not quantify exactly the accuracy of the retrieval technique. However, it appeared clearly that the obtained accuracy is good enough for the estimation of second-order statistical quantities, acceptable for third-order quantities and insufficient for fourth-order quantities. Therefore, the stereo technique in the present stage should not be thought as a self-contained universal tool to characterize the surface statistics. Instead, it should be used in conjunction with other well calibrated but sparse reference measurement (such as wave gauges) for crossvalidation and calibration. It then completes the statistical analysis in as much as it provides a snapshot of the threedimensional field and allows for the evaluation of higherorder spatial statistics.

[65] The proposed methodology is a first step before a systematic exploitation of more complete data sets. In particular forthcoming studies should be aimed at the analysis and parametrization of the directional structure functions of arbitrary orders that play an important role in the analytical scattering models for ocean remote sensing.

[66] Acknowledgments. The authors would like to thank B. Chapron (Ifremer, France) for useful remarks. Stereo data were obtained during joint experimental campaign with F. Ardhuin (Ifremer, France), A. Benetazo (ISMAR, Italy) and V. Kudryavtsev (NIERSC, Russian Federation). Authors are grateful for the help and support throughout the experiment. This work was supported by the ANR project ANR-09-BLAN-0232-01 SIMODE, also by Ukrainian State Agency of Science, Innovations and Information under contract M/412-2011, and by EU FP7/ ERC projects PBL-PMES (grant 227915) and COCONET (grant 287844).

\section{References}

Banner, M. (1990), Equilibrium spectra of wind waves, J. Phys. Oceanogr., 20(7), 966-984.

Banner, M., J. Trinder, and I. Jones (1989), Wavenumber spectra of short gravity waves, J. Fluid Mech., 198, 321-344.

Bechle, A. J., and C. H. Wu (2011), Virtual wave gauges based upon stereo imaging for measuring surface wave characteristics, Coastal Eng., 58 , $305-316$.

Benetazzo, A. (2006), Measurements of short water waves using stereo matched image sequences, Coastal Eng., 53(12), 1013-1032.

Benetazzo, A., F. Fedele, G. Gallego, P. Shih, and A. Yezzi (2012), Offshore stereo measurements of gravity waves, Coastal Eng., 64, 127-138. Bouguet, J. Y. (2004), Camera calibration toolbox for Matlab, technical report, Calif. Inst. of Technol., Pasadena. [Available at www.vision. caltech.edu/bouguetj/calib doc/index.html.]

Bréon, F., and N. Henriot (2006), Spaceborne observations of ocean glint reflectance and modeling of wave slope distributions, J. Geophys. Res., 111, C06005, doi:10.1029/2005JC003343.

Bringer, A., C.-A. Guérin, B. Chapron, and A. Mouche (2012), Peakedness effects in near-nadir radar observations of the sea surface, IEEE Trans. Geosci. Remote Sens., 50(9), 3293-3301.

Caulliez, G., and C.-A. Guérin (2012), Higher-order statistical analysis of short wind-waves, J. Geophys. Res., 117, C06002, doi:10.1029/ 2011JC007854.

Chen, K., A. Fung, and D. Weissman (1992), A backscattering model for ocean surfaces, IEEE Trans. Geosci. Remote Sens., 30(4), 811-817.

Chen, K., A. Fung, and F. Amar (1993), An empirical bispectrum model for sea surface scattering, IEEE Trans. Geosci. and Remote Sens., 31(4), $830-835$.

Cote, L., L. Davis, W. Marcs, R. McGough, E. Mehr, W. Pierson, I. Ropek, G. Stephenson, and R. Vetter (1960), The Directional Spectrum of Wind-
Generated Sea Waves as Determined From Data Obtained by the Stereo Wave Observation Project, N. Y. Univ., New York.

Cox, C., and W. Munk (1954), Statistics of the sea surface derived from sun glitter, J. Mar. Res., 13(2), 198-227.

Cox, C., and W. Munk (1956), Slopes of the sea surface deduced from photographs of sun glitter, Bull. Scripps Inst. Oceanogr., 6(9), 401-488. de Vries, S., D. Hill, M. de Schipper, and M. Stive (2011), Remote sensing of surf zone waves using stereo imaging, Coastal Eng., 58(3), 239-250. Doob, J. (1953), Stochastic Processes, Wiley, New York.

Fedele, F., A. Benetazzo, and G. Forristall (2011), Space-time waves and spectra in the northern adriatic sea via a wave acquisition stereo system, paper presented at 30th International Conference on Ocean, Offshore and Artic Engineering, ASME, Rotterdam, Netherlands.

Fedele, F., A. Benetazzo, G. Gallego Bonet, P. Shih, A. Yezzi, and F. Barbariol (2012), Wave statistics and space-time extremes via stereo imaging, paper presented at 22nd International Society of Offshore and Polar Engineers, Rhodes, Greece.

Gallego, G., A. Yezzi, F. Fedele, and A. Benetazzo (2011a), A variational stereo method for the three-dimensional reconstruction of ocean waves, IEEE Trans. Geosci. Remote Sens., 49(11), 4445-4457.

Gallego, G., A. Yezzi, F. Fedele, and A. Benetazzo (2011b), A variational wave acquisition stereo system for the $3-d$ reconstruction of oceanic sea states, paper presented at 30th International Conference on Ocean, Offshore and Artic Engineering, ASME, Rotterdam, Netherlands.

Grilli, S., C.-A. Guérin, and B. Goldstein (2011), Ocean wave reconstruction algorithms based on spatio-temporal data acquired by a flash lidar camera, paper presented at 22nd International Society of Offshore and Polar Engineers, Rhodes, Greece.

Holthuijsen, L. (1983), Observations of the directional distribution of ocean wave energy, J. Phys. Oceanogr., 13, 191-207.

Huang, N., S. Long, C. Tung, Y. Yuan, and L. Bliven (1983), A nongaussian statistical model for surface elevation of nonlinear random wave fields, J. Geophys. Res., 88(C12), 7597-7606.

Ishimaru, A. (1999), Wave Propagation and Scattering in Random Media, IEEE/OUP Ser. Electromagn. Wave Theory, vol. 12, IEEE, Piscataway, N. J.

Jähne, B., M. Schmidt, and R. Rocholz (2005), Combined optical slope/ height measurements of short wind waves: Principle and calibration, Meas. Sci. Technol., 16, 1937.

Kosnik, M., and V. Dulov (2011), Extraction of short wind wave spectra from stereo images of the sea surface, Meas. Sci. Technol., 22, 015504.

Longuet-Higgins, M., D. Cartwright, and N. Smith (1963), Observations of the directional spectrum of sea waves using the motions of a floating buoy, in Ocean Wave Spectra, pp. 111-136, Prentice-Hall, Easton, Md.

McDaniel, S. (2003), Microwave backscatter from non-gaussian seas, IEEE Trans. Geosci. Remote Sens., 41(1), 52-58.

Monin, A., and A. Yaglom (1999), Statistical Fluid Mechanics: The Mechanics of Turbulence, MIT Press, Cambridge, Mass.

Mouche, A., B. Chapron, N. Reul, D. Hauser, and Y. Quilfen (2007), Importance of the sea surface curvature to interpret the normalized radar cross section, J. Geophys. Res., 112, C10002, doi:10.1029/2006JC004010.

Shemdin, O., H. Tran, and S. Wu (1988), Directional measurement of short ocean waves with stereophotography, J. Geophys. Res., 93(C11), 13,891-13,901, doi:10.1029/JC093iC11p13891.

Tayfun, M., and F. Fedele (2007), Wave-height distributions and nonlinear effects, Ocean Eng., 34(11), 1631-1649.

Tsubaki, R., and I. Fujita (2005), Stereoscopic measurement of a fluctuating free surface with discontinuities, Meas. Sci. Technol., 16, 1894.

Vandemark, D., B. Chapron, J. Sun, G. Crescenti, and H. Graber (2004), Ocean wave slope observations using radar backscatter and laser altimeters, J. Phys. Oceanogr., 34, 2825-2842.

Voronovich, A. G. (1994), Wave Scattering From Rough Surfaces, Ser. Wave Phenom., vol. 17, Springer, Berlin.

Wanek, J., and C. Wu (2006), Automated trinocular stereo imaging system for threedimensional surface wave measurements, Ocean Eng., 33(5-6), 723-747.

Zappa, C., M. Banner, H. Schultz, A. Corrada-Emmanuel, L. Wolff, and J. Yalcin (2008), Retrieval of short ocean wave slope using polarimetric imaging, Meas. Sci. Technol., 19, 055503.

Zhang, X., and C. Cox (1994), Measuring the two-dimensional structure of a wavy water surface optically: A surface gradient detector, Exp. Fluids, $17,225-237$. 